\title{
MHD Analysis of Couple Stress Hybrid Nanofluid Free Stream over a Spinning Darcy-Forchheimer Porous Disc under the Effect of Thermal Radiation
}

\author{
Muhammad Zubair, ${ }^{1}$ Muhammad Jawad, ${ }^{2}$ Ebenezer Bonyah $\mathbb{D}^{3},{ }^{3}$ and Rashid Jan ${ }^{4}$ \\ ${ }^{1}$ Department of Mathematics, University of Peshawar, Peshawar, 25120 Khyber Pakhtunkhwa, Pakistan \\ ${ }^{2}$ Department of Mathematics, University of Swabi, Swabi, Khyber Pakhtunkhwa, Pakistan \\ ${ }^{3}$ Department of Mathematics Education, Akenten Appiah Menka University of Skills Training and Entrepreneurial Development, \\ Kumasi, Ghana \\ ${ }^{4}$ Department of Mathematics, Abdul Wali Khan University, Mardan, 23200 Khyber Pakhtunkhwa, Pakistan
}

Correspondence should be addressed to Ebenezer Bonyah; ebbonya@gmail.com

Received 5 July 2021; Revised 18 September 2021; Accepted 18 October 2021; Published 11 December 2021

Academic Editor: Waqar A. Khan

Copyright (c) 2021 Muhammad Zubair et al. This is an open access article distributed under the Creative Commons Attribution License, which permits unrestricted use, distribution, and reproduction in any medium, provided the original work is properly cited.

\begin{abstract}
This article develops the semianalytical analysis of couple stress hybrid nanofluid free stream past a rotating disc by applying the magnetic flux effects and radiation of thermal energy. The analysis of such kind of mixed convective flow is most important due to numerous industrial applications such as electronic devices, atomic reactors, central solar energy equipment, and heat transferring devices. The impact of variable permeability is also considered in the study. The permeability of the disc obeys the Darcy-Forchheimer model. The hybrid nanofluid is composed of water, titanium dioxide, and aluminum oxide. The set of governing equations in the PDE form are transformed to couple ODEs by applying similarity transformations. The ODE set are solved by applying the technique of HAM. The graphs of impacts of numerous physical parameters over momentum, energy, and concentration profiles are drawn in computer-based application Mathematica 11.0.1. In the sundry physical parameters, the porosity parameter, Reynolds number, inertial parameter, Prandtl number, Schmidt number, couple stress, and quotient of rotational momentum to elongating rate are included. During the analysis, it is found that the momentum profile of the couple stress hybrid nanofluid enhances with local inertial parameter, couple stress parameter, porosity parameter, and Reynolds number but declines for the growth in Hartmann number. Heat transfer rate enhances for radiation parameter but decreases in variable for temperature, thermal stratification parameter, thermophoresis parameter, and Brownian parameter.
\end{abstract}

\section{Introduction}

The era in which we are living the use of machinery and modern technology is too much like in civil, mechanical, and engineering. It is fact that production of heat is associated with machinery. The extra production of heat causes lower efficiency of the machinery and more consumption of energy. To reduce such production of useless heat in the running machinery, we used such technologies and approaches in which the use of such fluids is very common like water, mineral, organic (ethylene glycol, refrigerants, and tri-ethyl- ene-glycol), bio, and herbal oils. The uses of such fluids are in cement, lather, garments, tiles, and rock cutting industries. Such kind of fluids was used in scientific purposes like hydroelectric, thermal electric, and nuclear power stations. But the uses of such common fluids have some limitations, because these common fluids used as a coolant has low heat capacity. Metals (gold, silver, and copper), metal oxides (for example, aluminum oxide $\left(\mathrm{Al}_{2} \mathrm{O}_{3}\right)$, iron oxide $\left(\mathrm{Fe}_{2} \mathrm{O}_{3}\right)$, silver oxide $\left(\mathrm{Ag}_{2} \mathrm{O}\right)$, zirconia $\left(\mathrm{ZrO}_{2}\right)$, titania $\left(\mathrm{TiO}_{2}\right)$, alloy of metals, metal nitrides (AlN, SiN, and $\mathrm{SiN}$ ), and metal carbides (SiC) have high heat capacity as compared to the common fluids; thus, 
by the amalgamation of common fluids and metals, metal oxides and alloy of metals enhance the heat capacity of the common fluids. This concept was presented by the pioneer scientist Choi and Eastman [1]. At the beginning of invention of such new kind of suspension, the particle size in base fluids was in such a range that they were sediment after some time and its efficiency was not up to mark. So, after this, the researched recommended that the particle size should be smaller to enhance the heat capacity of the suspension and reduces the chances of sedimentation of the particles in the base fluids. When magnitude of metal particle inside the common liquid is in the range $\leq 20 \mathrm{~nm}$, then, the efficiency of such suspension is better. The solid particles are called nanoparticles, and common fluids in the suspensions are known as base fluids. Therefore, this new kind of suspension is called nanofluid. For the better performance of these nanofluids, the researchers add another kind of solid powder of nanosized particles. In the means due to hybridization of nanoparticles in the nanoliquid, the nanofluid heat capacity enhances. This is the next generation of the nanofluid. The size of the nanoparticles in the nanofluid or in the hybridnaofluid should be so small to flow through the porous medium [2]. Madhesh et al. [3] studied the experimental flow of heat in the hybrid nanofluid of $\mathrm{Cu}-\mathrm{TiO}_{2}$ across a tubular shaped container; the nanoparticles volume fraction during the experiment was $0.1 \%$ to $2 \%$. Senthilaraja et al. [4] studied experimentally the thermal conductivity of hybrid nanofluid composed of $\mathrm{Cu}-\mathrm{Al}_{2} \mathrm{O}_{3} /$ $\mathrm{H}_{2} \mathrm{O}$, during the experiment the nanoparticle volume fraction was $(0.05,0.1$, and $0.2 \%)$ and temperature variation was $20^{\circ} \mathrm{C}$ to $60^{\circ} \mathrm{C}$. Bhosale and Borse [5] experimentally showed that the hybrid nanofluid is better significantly than conventional fluid in critical heat flux (CHF) by using a Ni-Cr wire in pool boiling. He et al. [6] prepared a new kind of hybrid nanofluid composed of SWCNTs (single-wall carbon nanotubes) and CuSNPs (copper sulfide nanoparticles) into the base fluid PSS (styrene sulfonate) and analyze this new kind of hybrid nanofluid for production of electricity. Esfe et al. [7] experimentally analyzed the impact of nanoparticle percentage in the base fluid over the thermal conductivity of the hybrid nanofluid. Syam et al. [8] demonstrated experimentally the impact of MHD on the thermal conductivity and viscosity of hybrid nanofluid of $\mathrm{ND}-\mathrm{Co}_{3} \mathrm{O}_{4}$ (ND: nanodiamond). The shape of the nanoparticles like spherical, cylindrical, brick, platelet, or blade shape affects the thermal capacity of the nanofluid. The nanofluid emerged as a favorable heat transferring agent from last few decades of years due to contempt of inconsistency in the literature of the nanofluid and lacking of understanding of the heat transfer mechanisms. With regard to the extension of nanofluid study, the researchers invent a new kind of fluid by adding various kinds of nanoparticles in mixture/composite form in the conventional fluid; this type of fluid is identified by a hybrid nanofluid. As compared to the nanofluid, the hybrid nanofluid is the good heat transferring agent due to less disadvantages, enhanced thermal characteristics, and synergistic effect of nanoparticles of multikind of nanoparticles versus single kind of nanoparticle and less pressure drop characteristic. For the industrial use production of nanofluid, hybrid nanofluid is the great challenge for researchers due to selection of suitable nanomaterial which is affordable in cost and fulfills the required needs, long-term stability, and simple in production [9]. Haidera et al. [10] have investigated the stream of hybrid nanofluid across the medium obeying the Darcy-Forchheimer model. They solve this flow by the shooting method by keeping the slip condition of momentum as well as applying thermal radiation and stratification. Acharya et al. [11] have explored the results related to the MHD stream of nanofluid over a spinning disc under the inspirations of Hall current and thermal radiation. They solve the problem through the RK-4 approach and then compare their results with a shooting technique. Farooq et al. [12] have inspected the entropy production in the hybrid nanofluid over a stretched rotting circular disc. They investigated the influence of transportation and viscous effects. TD, $M G$, and Brinkman models are applied to entropy generation to find the effective thermal conductivity. Islam et al. [13] have scrutinized the flow of the Maxwell nanofluid on an elongating cylinder. For the best analysis, they apply the heat in the form of radiation; the fluid is also heated up due to joule heating. Zubair et al. [14] have explored the results of nanofluid flow inside a couple of spinning discs with Cattaneo-Cristove heat flux; the discs are porous following the Darcy-Forchheimer model. They also analyze the entropy production during the flow of the nanofluid. Khan et al. [15] have explored the stream of couple stress fluid past a stretched surface. This flow on the stretched sheet is due to the periodic oscillations. Hybrid nanofluid with mixed convection boundary layer stream has a lot of practical applications like in electronic devices as a coolant, solar receivers, nuclear reactors, and heat exchangers [16]. Due to these practical applications of mixed convection boundary layer stream, this is mandatory to deeply study its benefits and welfares. Hayat et al. [17] have scrutinized analytically the mixed convection boundary layer stream of the nanofluid past a stretched cylinder. Turkyilmazoglu [18] has examined the heat transfer in the flow of mixed convection nanofluid past a moving flat plate. Haroun et al. [19] have investigated the nanofluid stream over a stretching/shrinking belt with convective boundary condition and solved by applying spectral relaxation method. Mirmasoumi et al. [20] have investigated the nanofluid flow inside a cylindrical tube with convective boundary condition. Mahday [21] explored the numerical results about the heat transfer in the mixed convective boundary layer flow over a vertically standing stretched sheet due to variable thermal conductivity and viscosity. Vasumathi and Rao [22] investigated the laminar flow of mixed convective boundary under MHD past over the nonlinear stretched surface. Sumalatha and Shanker [23] studied the impact of Soret and Dufour effects on the mixed convective boundary layer stream under MHD past a nonuniform heated plate. Kumar et al. [24] explored the nanofluid flow in a vertical channel with robin boundary constrains. Shehzad et al. [25] investigated the viscous fluid flow by keeping interference of 
viscous dissipation and joule heating. Malvandi et al. [26] have considered the mixed convective flow of $\mathrm{Al}_{2} \mathrm{O}_{3}$ and water nanofluid annulus under the impact of the magnetic field. Selimefedigil et al. [27] have reported the result of their studies about the mixed convective flow of the nanofluid made up of $\mathrm{SiO}_{2}$ and $\mathrm{H}_{2} \mathrm{O}$ over a spinning cylinder. Hayat and Nadeem [28] studied the transfer of heat through the hybrid nanofluid composed of $\mathrm{Ag}, \mathrm{CuO}$ solid nanoparticles, and water; the impact of chemical reaction in the hybrid nanofluid is also encountered. Rashad et al. [29] have explored the outcomes of the hybrid nanofluid composed of $\mathrm{Cu}, \mathrm{Al}_{2} \mathrm{O}_{3}$, and water; natural convective flow under the impact of magnetic field; and joule heating. Usman et al. [30] investigated the impact of nonlinear thermal radiation over a time-dependent variable conductive hybrid nanofluid past a spinning plate. Mansour et al. [31] analyze the entropy generation in the MHD convective boundary flow inside a square-shaped cavity with heat source/sink. Sheikholeslami et al. [32] investigated the MHD flow of the hybrid nanofluid inside a round-shaped cavity with two heaters. Kumar and Sarkar [33] investigated the transfer of heat and drop of pressure of heat sink experimentally and present results numerically. Boulahia et al. [34] analyzed the entropy production ina convective boundary flow of the hybrid nanofluid inside a closed figure, wavy-shaped heat source placed at the center. Urmi et al. [35] explore experimentally the viscosity and thermal conductivity of hybridnaofluid $\mathrm{TiO}_{2}-\mathrm{A}$ $\mathrm{l}_{2} \mathrm{O}_{3}$, and ethylene glycol is used as the base fluid. The flow of convective fluid across the porous medium have a lot of practical applications like biomedical engineering, ecofriendly engineering, petroleum engineering, civil engineering, and agrarian sciences. By means of porous medium studies, we can calculate underground water pollution by toxic fluids, collect more and more data about gas and petroleum at underground reservoirs, water seepage in ground during drainage and irrigation, biosensors, to study the infrastructure of concrete construction, as well as in petrochemicals. For the study of these kinds of flows in the porous medium, we adopt Darcy's law to build a mathematical model for the flow. Darcy's law is applicable to the flow when the Reynolds number does not exceeds unity, but as the Reynolds number beats unity the pressure drops in the fluid due to inertial effects. Therefore, Forchheimer [36] introduce a nonlinear term in his work to calculate the inertial effects to the flow in permeable medium. Muskat [37] also reported their results about the Forchheimer term. Seddeek [38] investigated the mass and heat transfer through the mixed convective boundary hybrid nanofluid past an isothermal upstanding flat sheet. Sadiq and Hayat [39] studied the 2D MHD flow of the Maxwell nanofluid past a linearly elongating belt. Bakar et al. [40] analyzed the convective boundary layer flow near a stagnation point with slip condition past a DarcyForchheimer porous surface. Umavathi et al. [41] studied the natural convective stream inside an upstanding porous medium-filled rectangular duct. Hayat et al. [42] investigated the heat transfer in SWCNTs and MWCNTs over the Darcy-Forchheimer porous disc. Shah et al. [43] explored the 3D micropolar flow past a spinning Darcy-
Forchheimer porous disc. Sajjadi et al. [44] analyzed MRT, LBM, and MHD natural convective boundary flow across a porous medium. Muhammad et al. [45] demonstrated the flow of a viscous nanofluid past a curved sheet stretched nonlinearly with the Darcy-Forchheimer model. Sadiq et al. [46] illustrated impacts of slip conditions (thermal and velocity) over the heat transfer in a nanofluid past a Darcy-Forchheimer porous spinning disc. Alghamdi et al. [47] examined the transfer of heat in the Casson biphasic nanofluid through the Darcy-Brinkman porous medium. Khan et al. [48] explored the transfer of heat and mass in a nonaxisymmetric flow over a linearly stretched sheet. Rasool et al. [49] investigated numerically the MHD Darcy-Forchheimer flow of nanofluid over a nonlinear stretched sheet. Waqas et al. [50] investigated numerically the Darcy-Forchheimer flow of couple stress bioconvective nanofluid past an inclined porous stretched cylinder.

Now, researchers take interest to analyze the nanofluid flow across the medium having variable permeability and porosity. By keeping in mind the above study, we examined the hybrid couple stress nanofluid across the medium having variable permeability and porosity under the impact of MHD. In this study, the rotation of the medium having variable permeability and porosity was also encountered.

\section{Mathematical Formulation}

We assumed the 3D stream of hybrid nanofluid past a rotating circular disc. The angular momentum of the disc is represented by $\Omega$. The disc is made up of a porous medium obeying the Darcy-Forchheimer model. The permeability and porosity of the circular disc are variable. This flow of the couple stress hybrid nanofluid is in a steady state. The fluid is electrically conducted and heated up by applying thermal radiation which is also affected by the magnetic field. The electrical conductivity of the hybrid nanofluid is symbolized by $\sigma_{\text {hnf }}$. The flow of the couple stress fluid past a rotating disc is represented geometrically as below.

Here, $(u, v, w)$ are the momentum components taken in the direction of $(r, \psi, z)$. The effect of concentration is also encountered in the study. The principal equations of the assumed stream are as follows:

$$
\begin{aligned}
& \frac{\partial u}{\partial r}+\frac{u}{r}+\frac{\partial w}{\partial z}=0 \\
& u \frac{\partial u}{\partial r}-\frac{v^{2}}{r}+w \frac{\partial u}{\partial z}=v_{\mathrm{hnf}}\left(\frac{\partial^{2} u}{\partial r^{2}}+\frac{1}{r} \frac{\partial u}{\partial r}-\frac{u}{r^{2}}+\frac{\partial^{2} u}{\partial z^{2}}\right) \\
& -\frac{v_{\mathrm{hnf}} \varepsilon(z)}{K(z)} u-\frac{\sigma_{\mathrm{hnf}} \beta_{0}^{2}}{\rho_{\mathrm{hnf}}} u-\frac{\eta_{0}}{\rho_{\mathrm{hnf}}} \frac{\partial^{4} u}{\partial z^{4}} \\
& -\frac{C_{b} \varepsilon^{2}(z)}{\sqrt{K(z)}} u \sqrt{u^{2}+v^{2}},
\end{aligned}
$$




$$
\begin{aligned}
& u \frac{\partial v}{\partial r}+\frac{u v^{2}}{r}+w \frac{\partial u}{\partial z}=v_{\mathrm{hnf}}\left(\frac{\partial^{2} v}{\partial r^{2}}+\frac{1}{r} \frac{\partial v}{\partial r}-\frac{v}{r^{2}}+\frac{\partial^{2} v}{\partial z^{2}}\right) \\
& -\frac{v_{\mathrm{hnf}} \varepsilon(z)}{K(z)} v-\frac{\sigma_{\mathrm{hnf}} \beta_{0}^{2}}{\rho_{\mathrm{hnf}}} v-\frac{\eta_{0}}{\rho_{\mathrm{hnf}}} \frac{\partial^{4} v}{\partial z^{4}} \\
& -\frac{C_{b} \varepsilon^{2}(z)}{\sqrt{K(z)}} v \sqrt{u^{2}+v^{2}} \\
& u \frac{\partial w}{\partial r}+w \frac{\partial w}{\partial z}=v_{\mathrm{hnf}}\left(\frac{\partial^{2} w}{\partial r^{2}}+\frac{1}{r} \frac{\partial w}{\partial r}+\frac{\partial^{2} w}{\partial z^{2}}\right)-\frac{v_{\mathrm{hnf}} \varepsilon(z)}{K(z)} \\
& \cdot w-\frac{\sigma_{\mathrm{hnf}} \beta_{0}^{2}}{\rho_{\mathrm{hnf}}} w-\frac{\eta_{0}}{\rho_{\mathrm{hnf}}} \frac{\partial^{4} w}{\partial z^{4}} \\
& -\frac{C_{b} \varepsilon^{2}(z)}{\sqrt{K(z)}} w \sqrt{u^{2}+v^{2}} \\
& u \frac{\partial T}{\partial r}+w \frac{\partial T}{\partial z}=\alpha_{\text {hnf }}\left(\frac{\partial^{2} T}{\partial r^{2}}+\frac{1}{r} \frac{\partial T}{\partial r}+\frac{\partial^{2} T}{\partial z^{2}}\right) \\
& +\tau_{\mathrm{hnf}}\left[D_{B}\left(C_{z} T_{z}\right)+\frac{D_{T}}{T_{\infty}}\left(T_{z}\right)^{2}\right] \\
& -\frac{1}{(\rho c p)_{\mathrm{hnf}}} \frac{16 \sigma^{*} T_{\infty}^{3}}{3 k} \frac{\partial^{2} T}{\partial z^{2}} \\
& u \frac{\partial C}{\partial r}+v \frac{\partial C}{\partial z}=D_{B} \frac{\partial^{2} C}{\partial z^{2}}+\left(\frac{D_{T}}{T_{\infty}}\right) \frac{\partial^{2} T}{\partial z^{2}} .
\end{aligned}
$$

The variable permeability and porosity of the disc are given in the mathematical form as

$$
\begin{aligned}
& K(z)=K_{\infty}\left(1+d e^{-z / r}\right), \\
& \varepsilon(z)=\varepsilon_{\infty}\left(1+d^{*} e^{-z / r}\right) .
\end{aligned}
$$

The boundary conditions for the problem are

$u=L \frac{\partial u}{\partial z}, \quad v=r \Omega+L \frac{\partial u}{\partial z}, \quad w=0, \quad T=T_{w}, \quad C=C_{w}, \quad$ at $z=0$, $u \longrightarrow 0, \quad v \longrightarrow 0, \quad T \longrightarrow T_{\infty}, \quad C \longrightarrow C_{w}$, at $z \longrightarrow \infty$.

The similarity transformation selected for the problem to transform the governing PDEs given in equation (1) to (6) to ODEs is

$$
\begin{gathered}
u=r \Omega f^{\prime}(\xi), v=r \Omega g(\xi), w=-\sqrt{2 \Omega v_{f}} f(\xi), \\
\theta(\xi)=\frac{T-T_{\infty}}{T_{w}-T_{0}}, \phi(\xi)=\frac{C-C_{\infty}}{C_{w}-C_{0}}, \text { Here } \xi=\sqrt{\frac{2 \Omega}{v_{f}}} z .
\end{gathered}
$$

The theoretical mathematical model for the hybrid nanofluid [12] applies to governing equations (2) to (6):

$$
\left.\begin{array}{rl}
\mu_{\mathrm{hnf}}= & \frac{\mu_{f}}{\left(1-\phi_{1}-\phi_{2}\right)^{2.5}}, v_{\mathrm{hnf}}=\frac{\mu_{\mathrm{hnf}}}{\rho_{\mathrm{hnf}}}, \rho_{\mathrm{hnf}}=\rho_{f}\left(1-\phi_{1}-\phi_{2}\right)+\rho_{1} \phi_{1}+\rho_{2} \phi_{2}, \\
\alpha_{\mathrm{hnf}}= & \frac{\kappa_{\mathrm{hnf}}}{\left(\rho c_{p}\right)_{\mathrm{hnf}}},\left(\rho c_{p}\right)_{\mathrm{hnf}}=\left(\rho c_{p}\right)_{f}\left(1-\phi_{1}-\phi_{2}\right)+\left(\rho c_{p}\right)_{1} \phi_{1}+\left(\rho c_{p}\right)_{2} \phi_{2}, \\
\frac{\kappa_{\mathrm{hnf}}}{\kappa_{\mathrm{bf}}}= & \frac{\phi_{1} \kappa_{1}+\phi_{2} \kappa_{2}+2 \phi_{f} \kappa_{f}+2 \phi_{f}\left(\phi_{1} \kappa_{1}+\phi_{2} \kappa_{2}\right)-2\left(\phi_{1}+\phi_{2}\right)^{2} \kappa_{f}}{\phi_{1} \kappa_{1}+\phi_{2} \kappa_{2}+2 \phi_{f} \kappa_{f}-\phi_{f}\left(\phi_{1} \kappa_{1}+\phi_{2} \kappa_{2}\right)+\left(\phi_{1}+\phi_{2}\right)^{2} \kappa_{f}}, \\
\frac{\sigma_{\mathrm{hnf}}}{\sigma_{\mathrm{bf}}}= & \frac{\sigma_{2}+2 \sigma_{b f}-2 \phi_{2}\left(\sigma_{b f}-\sigma_{2}\right)}{\sigma_{2}+2 \sigma_{b f}+\phi_{2}\left(\sigma_{b f}-\sigma_{2}\right)}, \text { where } \frac{\sigma_{\mathrm{bf}}}{\sigma f}=\frac{\sigma_{1}+2 \sigma_{f}-2 \phi_{1}\left(\sigma_{f}-\sigma_{1}\right)}{\sigma_{1}+2 \sigma_{f}-2 \phi_{1}\left(\sigma_{f}-\sigma_{1}\right)} .
\end{array}\right\},
$$

$$
\begin{aligned}
& \frac{1}{\left(1-\phi_{1}-\phi_{2}\right)\left(1-\phi_{1}-\phi_{2}+\left(\left(\rho c_{p}\right)_{1} /\left(\rho c_{p}\right)_{f}\right) \phi_{1}+\left(\left(\rho c_{p}\right)_{2} /\left(\rho c_{p}\right)_{f}\right) \phi_{2}\right)} \\
& \cdot\left[\left[\frac{k_{\mathrm{hnf}}}{k_{f}}+\frac{4}{3} R\left(\frac{\theta}{\theta_{w}+s_{t}}+1\right)^{3}\right] \theta^{\prime}\right], \\
& +\operatorname{Pr} f \theta^{\prime}+\frac{\left(\rho c_{p}\right)_{\mathrm{hnp}} /\left(\rho c_{p}\right)_{p}}{\left(\rho c_{p}\right)_{\mathrm{hnf}} /\left(\rho c_{p}\right)_{f}}\left[N_{b} \theta^{\prime} \phi^{\prime}+N_{t} \theta^{\prime 2}\right]=0,
\end{aligned}
$$

$$
\phi^{\prime \prime}-\operatorname{Pr} \operatorname{Scf} \phi^{\prime}+\frac{N_{t}}{N_{b}} \theta^{\prime \prime}=0,
$$

$f=0, \quad f^{\prime}=\dot{\gamma} f^{\prime \prime}, \quad g=1+\gamma g^{\prime}, \quad \theta=1-s_{t}, \quad \phi=1, \quad$ at $\quad \xi=0$, $f^{\prime} \longrightarrow 0, \quad g \longrightarrow 0, \quad \theta \longrightarrow 0, \quad \phi \longrightarrow 0, \quad$ at $\quad \xi=\infty$.

$\tau\left(=\left(\rho c_{p}\right)_{s} /\left(\rho c_{p}\right)_{f}\right)$ represents the quotient of heat capacity of solid nanoparticles to base fluid heat capacity, $\alpha_{\mathrm{hnf}}(=$ $\left.\kappa_{\mathrm{hnf}} /\left(\rho c_{p}\right)_{\mathrm{hnf}}\right)$ is ratio of thermal conductivity to specific heat of the hybrid nanofluid, $M\left(=\sigma B_{0}^{2} / b \rho_{f}\right)$ represents Hartmann number, $\alpha\left(=L \sqrt{2 \Omega / v_{f}}\right)$ indicates the velocity slip parameter with $L$ indicating the velocity slip coefficient, $\lambda\left(=K_{\infty} / r^{2} \varepsilon_{\infty}\right)$ 
shows porosity parameter, $K=\eta_{0} b / \rho v_{f}$ symbolizes the parameter of couple stress, $\operatorname{Re}\left(=U_{w} r / v_{f}\right)$ represents local Reynolds number, $\operatorname{Fr}\left(=C_{b} \varepsilon_{\infty}^{2} r / \sqrt{K_{\infty}}\right)$ expresses local inertial parameter with $K_{\infty}$ permeability and $\varepsilon_{\infty}$ porosity, $\gamma\left(=\sqrt{\alpha / r}\left(P e_{t}^{1 / 2} / r\right)\right)$ is the parameter where $\mathrm{P} e_{t}=\mathrm{Re} \cdot \mathrm{Pr}$, $R\left(=4 \sigma^{*} T_{\infty}^{3} / k \kappa_{f}\right)$ represents the radiation parameter, $\theta_{w}($ $\left.=T_{0} /(A \cdot r)\right)$ is the temperature variable, $s_{t}(=B / A)$ is thermal stratification parameter with $A$ and $B$ any dimensional constants, $D_{B}$ indicates Brownian diffusion coefficient, $D_{T}$ demonstrates the coefficient of thermophoretic diffusion, $\mathrm{Nt}=\left(\left(\rho c_{p}\right)_{s} D_{T}\left(T_{w}-T_{\infty}\right)\right) /\left(\left(\rho c_{p}\right)_{f} T_{\infty} v_{f}\right)$ indicates thermophoresis parameter, and $\mathrm{Nb}=\left(\left(\rho c_{p}\right)_{s} D_{B}\left(C_{w}-C_{\infty}\right)\right) /($ $\left.\left(\rho c_{p}\right)_{f} v_{f}\right)$ is the Brownian motion parameter.

\section{Thermophysical Properties}

Thermophysical properties are the properties of the material which is considered very important in the transfer of heat. The thermophysical properties of a substance comprises specific heat capacity $\left(\rho c_{p}\right)$, thermal conductivity $\kappa$, coefficient of liner and volumetric expansion $\alpha_{t}$ and $\beta_{t}$, heat of vapori-

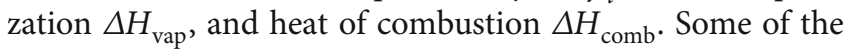
thermophysical properties of titanium dioxide $\mathrm{TiO}_{2}$, aluminum oxide $\mathrm{Al}_{2} \mathrm{O}_{3}$, and water $\mathrm{H}_{2} \mathrm{O}$ related to our problem are given in the table given below.

\section{Engineer Interest Quantities}

In the modern era, the use of mechanical and electrical equipment is much more; the generation of heat and friction must be associated with running machineries. The production of useless heat and friction reduces the efficiency of equipment. For the reduction of friction and transfer of useless heat, nanofluids are used in the equipment and machineries. For the flow analysis of couple stress hybrid nanofluid through permeable medium, the two terms skin friction and Nusselt number are more important. Skin friction is important to study the friction among the fluid and solid parts of the equipment, and the Nusselt number is calculated to examine the heat transfer rate through fluids. These two terms are explained below.

4.1. Skin Friction. The skin friction for the current phenomenon is defined mathematically in the nondimensional differential form as follows:

$$
\left.\begin{array}{l}
(\mathrm{Re})^{1 / 2} C_{f}=\frac{1}{\left(1-\phi_{1}-\phi_{2}\right)^{2.5}} f^{\prime \prime}(0), \\
(\mathrm{Re})^{1 / 2} C_{g}=\frac{1}{\left(1-\phi_{1}-\phi_{2}\right)^{2.5}} g^{\prime}(0),
\end{array}\right\}
$$

4.2. Local Nusselt Constant. The Nusselt number for the current problem is defined mathematically in the nondimen- sional differential form as follows:

$$
\frac{1}{2}(\mathrm{Re})^{1 / 2} \mathrm{Nu}=-\left(\frac{\kappa_{\mathrm{hnf}}}{\kappa_{f}}+\frac{4}{3} \mathrm{Rd}\left(\frac{1}{\theta_{w}+s_{t}}+1\right)^{3}\right) \theta^{\prime}(0)
$$

\section{Solution by HAM}

The semianalytic technique HAM is applied to our modeled problem given in equations (12)-(15) by utilizing the boundary conditions in equation (16). The schematic representation of the HAM technique is as follows.

The linear operators $L_{\widehat{f}}, L_{\widehat{g}}, L_{\widehat{\theta}}$, and $L_{\widehat{\phi}}$ for the HAM are demarcated as

$$
L_{\widehat{f}}(\widehat{f})=\widehat{f}^{v}, L_{\widehat{g}}(\widehat{g})=\widehat{g}^{\prime \prime}, L_{\widehat{\theta}}(\widehat{\theta})=\widehat{\theta}^{\prime \prime}, L_{\widehat{\phi}}(\widehat{\phi})=\widehat{\phi}^{\prime \prime}
$$

The nonlinear operators $N_{\widehat{f}}, N_{\widehat{g}}, N_{\widehat{\theta}}$, and $N_{\widehat{\phi}}$ for the HAM are defined as follows:

$$
\begin{aligned}
& N_{\widehat{f}}[\widehat{f}(\xi ; p), \widehat{g}(\xi ; p)]=\frac{1}{\left(1-\phi_{1}-\phi_{2}\right)\left(1-\phi_{1}-\phi_{2}+\left(\rho_{1} / \rho_{f}\right) \phi_{1}+\left(\rho_{2} / \rho_{f}\right) \phi_{2}\right)} \\
& \cdot\left(2 \widehat{f}_{\xi \xi \xi}-\frac{1}{2 \lambda \operatorname{Re}_{r}}\left(\frac{1+d^{*} e^{-\xi}}{1+d e^{-\xi}}\right) \widehat{f}_{\xi}\right)-F_{r}\left(\frac{1+d^{*} e^{-\xi}}{\sqrt{1+d e^{-\xi}}}\right) \\
& \cdot\left(\widehat{f}_{\xi}^{2}+\frac{1}{2} \widehat{g}^{2}\right)-\widehat{f}_{\xi}^{2}+\widehat{g}^{2}+2 \widehat{f} \widehat{f}_{\xi \xi} \\
& \left.-\frac{\sigma_{\mathrm{hnf}} / \sigma_{f}}{\rho_{\mathrm{hnf}} / \rho_{f}} M \widehat{f}_{\xi}+4 \frac{\rho_{f}}{\rho_{\mathrm{hnf}}} K \widehat{f}_{\xi \xi \xi \xi \xi \xi}\right\},
\end{aligned}
$$

$$
\begin{aligned}
& N_{\widehat{g}}[\widehat{f}(\xi ; p), \widehat{g}(\xi ; p)]=\frac{1}{\left(1-\phi_{1}-\phi_{2}\right)\left(1-\phi_{1}-\phi_{2}+\left(\rho_{1} / \rho_{f}\right) \phi_{1}+\left(\rho_{2} / \rho_{f}\right) \phi_{2}\right)} \\
& \cdot\left(2 \widehat{g}_{\xi \xi}-\frac{1}{2 \lambda \operatorname{Re}_{r}}\left(\frac{1+d^{*} e^{-\xi}}{1+d e^{-\xi}}\right) \widehat{g}\right)-F_{r}\left(\frac{1+d^{*} e^{-\xi}}{\sqrt{1+d e^{-\xi}}}\right)\left(\widehat{g}^{2}+\frac{1}{2} \widehat{f}_{\xi}^{2}\right) \\
& \left.-\widehat{f}_{\xi} \widehat{g}+\widehat{f} \widehat{g}_{\xi}-\frac{\sigma_{\mathrm{hnf}} / \sigma_{f}}{\rho_{\mathrm{hnf}} / \rho_{f}} M \widehat{g}+4 \frac{\rho_{f}}{\rho_{\mathrm{hnf}}} K \widehat{f}_{\xi \xi \xi \xi \xi}\right\},
\end{aligned}
$$

$$
\begin{aligned}
& N_{\widehat{\theta}}[\widehat{f}(\xi ; p), \widehat{\theta}(\xi ; p), \widehat{\phi}(\xi ; p)] \\
& =\frac{1}{\left(1-\phi_{1}-\phi_{2}\right)\left(1-\phi_{1}-\phi_{2}+\left(\left(\rho_{c p}\right)_{1} /\left(\rho_{c p}\right)_{f}\right) \phi_{1}+\left(\left(\rho_{c p}\right)_{2} /\left(\rho_{c p}\right)_{f}\right) \phi_{2}\right)} \\
& \quad \cdot\left[\left[\frac{k_{\mathrm{hnf}}}{k_{f}}+\frac{4}{3} R\left(\frac{\hat{\theta}}{\theta_{w}+s_{t}}+1\right)^{3}\right] \widehat{\theta}_{\xi}\right], \\
& +\operatorname{Pr} \widehat{f} \widehat{\theta}_{\xi}+\frac{\left(\rho_{c p}\right)_{\mathrm{hnp}} /\left(\rho_{c p}\right)_{p}}{\left(\rho_{c p}\right)_{\mathrm{hnf}} /\left(\rho_{c p}\right)_{f}}\left[N_{b} \widehat{\theta}_{\xi} \widehat{\phi}_{\xi}+N_{t} \widehat{\theta}_{\xi}^{2}\right],
\end{aligned}
$$

$$
N_{\widehat{\phi}}[\widehat{f}(\xi ; p), \widehat{\theta}(\xi ; p), \widehat{\phi}(\xi ; p)]=\widehat{\phi}_{\xi \xi}-\operatorname{Pr} \operatorname{Scf} \widehat{\phi}_{\xi}+\frac{N_{t}}{N_{b}} \widehat{\theta}_{\xi \xi}
$$


The $0^{\text {th }}$ order solution is as follows:

$$
\begin{aligned}
& (1-p) L_{\widehat{f}}\left[\widehat{f}(\xi ; p)-\widehat{f}_{0}\right]=p \hbar N_{\widehat{f}}[\widehat{f}(\xi ; p), \widehat{g}(\xi ; p)], \\
& (1-p) L_{\widehat{g}}\left[\widehat{g}(\xi ; p)-\widehat{g}_{0}\right]=p \hbar N_{\widehat{g}}[\widehat{f}(\xi ; p), \widehat{g}(\xi ; p)], \\
& (1-p) L_{\widehat{\theta}}\left[\widehat{\theta}(\xi ; p)-\widehat{\theta}_{0}\right]=p \hbar N_{\widehat{\theta}}[\widehat{f}(\xi ; p), \widehat{g}(\xi ; p) ; \widehat{\phi}(\xi ; p)], \\
& (1-p) L_{\widehat{\phi}}\left[\widehat{\phi}(\xi ; p)-\widehat{\phi}_{0}\right]=p \hbar N_{\widehat{\phi}}[\widehat{f}(\xi ; p), \widehat{g}(\xi ; p) ; \widehat{\phi}(\xi ; p)] .
\end{aligned}
$$

The conditions at the boundary point for the current phenomenon are transformed to nondimensional form as follows:

$$
\begin{aligned}
\left.\widehat{f}(\xi ; p)\right|_{\xi=0} & =0,\left.\frac{\partial}{\partial \xi} \widehat{f}(\xi ; p)\right|_{\xi=0}=\left.\gamma \frac{\partial^{2}}{\partial \xi^{2}} \widehat{f}(\xi ; p)\right|_{\xi=0},\left.\widehat{\theta}(\xi ; p)\right|_{\xi=0} \\
& =1-s t,\left.\widehat{\phi}(\xi ; p)\right|_{\xi=0}=1 \\
\left.\widehat{f}(\xi ; p)\right|_{\xi=\infty} & \longrightarrow 0,\left.\widehat{g}(\xi ; p)\right|_{\xi=\infty} \longrightarrow 0,\left.\widehat{\theta}(\xi ; p)\right|_{\xi=\infty} \\
& \longrightarrow 0,\left.\widehat{\phi}(\xi ; p)\right|_{\xi=0} \longrightarrow 0
\end{aligned}
$$

In the above system, " $p$ " represents the imbedding parameter lies in the rang $0 \leq p \leq 1$. To regulate the solution convergence for the problem, we choose the parameters $h_{\hat{f}}, h_{\widehat{g}}, h_{\hat{\theta}}$, and $h_{\hat{\phi}}$ in a best range. At the extreme limits of the embedding parameters " $p$ " that is $p=0$ and $p=1$, the problem solution reduces to the expressions as

$\widehat{f}(\xi ; 0)=\widehat{f}_{0}(\xi), \widehat{g}(\xi ; 0)=\widehat{g}_{0}(\xi), \widehat{\theta}(\xi ; 0)=\widehat{\theta}_{0}(\xi), \widehat{\phi}(\xi ; 0)=\widehat{\phi}_{0}(\xi)$,

Expand the functions $\widehat{f}(\xi ; p), \widehat{g}(\xi ; p), \widehat{\theta}(\xi ; p)$, and $\widehat{\phi}$ $(\xi ; p)$ by using the Taylor's series.

$f(\xi ; p)=f_{0}(\xi)+\sum_{j=1}^{\infty} f_{j}(\xi) p^{j}, \quad g(\xi ; p)=g_{0}(\xi)+\sum_{j=1}^{\infty} g_{j}(\xi) p^{j}$,

$\theta(\xi ; p)=\theta_{0}(\xi)+\sum_{j=1}^{\infty} \theta_{j}(\xi) p^{j}, \quad \phi(\xi ; p)=\phi_{0}(\xi)+\sum_{j=1}^{\infty} \phi_{j}(\xi) p^{j}$,

Where

$$
\begin{aligned}
& f_{j}(\xi)=\left.\frac{1}{j !} \frac{\partial}{\partial \xi} f(\xi ; p)\right|_{p=0}, \quad g_{j}(\xi)=\left.\frac{1}{j !} \frac{\partial}{\partial \xi} g(\xi ; p)\right|_{p=0}, \\
& \theta_{j}(\xi)=\left.\frac{1}{j !} \frac{\partial}{\partial \xi} \theta(\xi ; p)\right|_{p=0}, \quad \phi_{j}(\xi)=\left.\frac{1}{j !} \frac{\partial}{\partial \xi} \phi(\xi ; p)\right|_{p=0} .
\end{aligned}
$$

The $j^{\text {th }}$ order solution for the current problem is

$$
\begin{aligned}
& L_{\widehat{f}}\left[\widehat{f}_{j}(\xi)-\chi_{j} \widehat{f}_{j-1}(\xi)\right]=\hbar_{f} \mathfrak{R}_{j}^{\widehat{f}}(\xi), \\
& L_{\widehat{g}}\left[\widehat{g}_{j}(\xi)-\chi_{j} \widehat{g}_{j-1}(\xi)\right]=\hbar_{\widehat{g}} \mathfrak{R}_{j}^{\widehat{g}}(\xi), \\
& L_{\widehat{\theta}}\left[\widehat{\theta}_{j}(\xi)-\chi_{j} \widehat{\theta}_{j-1}(\xi)\right]=\hbar_{\hat{\theta}} \mathfrak{R}_{j}^{\widehat{\theta}}(\xi), \\
& L_{\widehat{\phi}}\left[\widehat{\phi}_{j}(\xi)-\chi_{j} \widehat{\phi}_{j-1}(\xi)\right]=\hbar_{\widehat{\phi}} \mathfrak{R}_{j}^{\widehat{\phi}}(\xi) .
\end{aligned}
$$

Here,

$$
\begin{aligned}
\mathfrak{R}_{j}^{\widehat{f}}(\xi)= & \frac{1}{\left(1-\phi_{1}-\phi_{2}\right)\left(1-\phi_{1}-\phi_{2}+\left(\rho_{1} / \rho_{f}\right) \phi_{1}+\left(\rho_{2} / \rho_{f}\right) \phi_{2}\right)} \\
& \cdot\left(2 \widehat{f}_{r}^{\prime}-\frac{1}{2 \lambda \operatorname{Re}_{r}}\left(\frac{1+d^{*} e^{-\xi}}{1+d e^{-\xi}}\right) \widehat{f}_{r}^{\prime}\right)-F_{r}\left(\frac{1+d^{*} e^{-\xi}}{\sqrt{1+d e^{-\xi}}}\right) \\
& \cdot\left(\sum_{r=0}^{j-1} \widehat{f}_{j-1-r}^{\prime} \widehat{f}_{r}^{\prime}+\frac{1}{2} \sum_{r=0}^{j-1} \widehat{g}_{j-1-r} \widehat{g}_{r}\right)-\sum_{r=0}^{j-1} \widehat{f}_{j-1-r}^{\prime} \widehat{f}_{r}^{\prime} \\
& +\sum_{r=0}^{j-1} \widehat{g}_{j-1-r} \widehat{g}_{r}+2 \sum_{r=0}^{j-1} \widehat{f}_{j-1-r} \widehat{f}_{r}^{\prime}-\frac{\sigma_{\mathrm{hnf}} / \sigma_{f}}{\rho_{\mathrm{hnf}} / \rho_{f}} \\
& \cdot M \widehat{f}_{r}^{\prime}+4 \frac{\rho_{f}}{\rho_{\mathrm{hnf}}} \widehat{f}_{r}^{\prime \prime},
\end{aligned}
$$

$$
\begin{aligned}
\mathfrak{R}_{j}^{\widehat{g}}(\xi)= & \frac{1}{\left(1-\phi_{1}-\phi_{2}\right)\left(1-\phi_{1}-\phi_{2}+\left(\rho_{1} / \rho_{f}\right) \phi_{1}+\left(\rho_{2} / \rho_{f}\right) \phi_{2}\right)} \\
& \cdot\left(2 \sum_{r=0}^{j-1} \widehat{g}_{j-1-r}^{\prime} \widehat{g}_{r}^{\prime}-\frac{1}{2 \lambda \operatorname{Re}_{r}}\left(\frac{1+d^{*} e^{-\xi}}{1+d e^{-\xi}}\right) \widehat{g}_{r}\right) \\
& -F_{r}\left(\frac{1+d^{*} e^{-\xi}}{\sqrt{1+d e^{-\xi}}}\right)\left(\sum_{r=0}^{j-1} \widehat{g}_{j-1-r} \widehat{g}_{r}+\frac{1}{2} \sum_{r=0}^{j-1} \widehat{f}_{j-1-r} \widehat{f}_{r}^{\prime}\right) \\
& -\sum_{r=0}^{j-1} \widehat{f}_{j-1-r}^{\prime} \widehat{g}_{r}+\sum_{r=0}^{j-1} \widehat{f}_{j-1-r}^{\prime} \widehat{g}_{r}^{\prime}-\frac{\sigma_{\mathrm{hnf}} / \sigma_{f}}{\rho_{\mathrm{hnf}} / \rho_{f}} M \widehat{g}_{r} \\
& +4 \frac{\rho_{f}}{\rho_{\mathrm{hnf}}} \widehat{K}_{f_{r}}^{\prime \prime \prime \prime \prime \prime},
\end{aligned}
$$

$$
\begin{aligned}
\mathfrak{R}_{j}^{\widehat{\theta}}(\xi)= & \frac{1}{\left(1-\phi_{1}-\phi_{2}\right)\left(1-\phi_{1}-\phi_{2}+\left(\left(\rho_{c p}\right)_{1}^{\prime}\left(\rho_{c p}\right)_{f}\right) \phi_{1}+\left(\left(\rho_{c p}\right)_{2} /\left(\rho_{c p}\right)_{f}\right) \phi_{2}\right)} \\
& \cdot\left[\left[\frac{k_{\mathrm{hnf}}}{k_{f}}+\frac{4}{3} R\left(\frac{\hat{\theta}_{r}}{\theta_{w}+s_{t}}+1\right)^{3}\right] \widehat{\theta}_{r}^{\prime}\right]{ }^{\prime}+\operatorname{Pr} \sum_{r=0}^{j-1} \widehat{f}_{j-1-r} \widehat{\theta}_{r}^{\prime} \\
& +\frac{\left(\rho_{c p}\right)_{\mathrm{hnp}} /\left(\rho_{c p}\right)_{p}}{\left(\rho_{c p}\right)_{\mathrm{hnf}} l\left(\rho_{c p}\right)_{f}}\left[\mathrm{Nb} \sum_{r=0}^{j-1} \widehat{\theta}_{j-1-r}^{\prime} \widehat{\phi}_{r}^{\prime}+\mathrm{Nt} \sum_{r=0}^{j-1} \widehat{\theta}_{j-1-r}^{\prime} \widehat{\theta}_{r}^{\prime}\right], \\
& \mathfrak{R}_{j}^{\phi}(\xi)=\widehat{\phi}_{r}^{\prime}-\operatorname{Pr} S c \sum_{r=0}^{j-1} \widehat{f}_{j-1-r} \widehat{\phi}_{r}^{\prime}+\frac{N_{t}}{N_{b}} \widehat{\theta}_{r}^{\prime} .
\end{aligned}
$$




\section{Result and Discussion}

Figure 1 shows the physical flow of the couple stress fluid past a rotatory disc. Figures 2-6 show the momentum profile $f(\eta)$ for various physical parameters, while Figures 7-9 demonstrate the impact of diverse physical parameters over the momentum profile $g(\eta)$. Figures $10-13$ are the representation of concentration against different physical parameters. The impressions of novel physical parameters on the energy profile of the couple stress nanofluid are represented in Figures 14-20. Figure 2 indicates the effect of local inertial parameter $\mathrm{Fr}$ on the momentum profile $f(\eta)$. Figure 2 indicates that the momentum profile decreases by enhancing the local inertial parameter Fr. Physically the increase in inertial parameter the increase in resistance in medium means decrease in the permeability of the medium as a result the decrease in momentum of the fluid. Figure 3 displays the impact of couple stress parameter $K$ over the momentum profile $f(\eta)$. This Figure 3 demonstrates that momentum profile boosts with the escalation of couple stress parameter. Physically the increase in couple stress parameter means the decrease in viscosity of the fluid so it is obvious that nonviscous fluid moves rapidly. Figure 4 portrays the relation of porosity parameter $\lambda$ versus momentum profile $f(\eta)$ of the couple stress nanofluid. Figure 4 indicates that there is direct relation of porosity parameter $\lambda$ with the momentum profile $f(\eta)$ of the couple stress nanofluid. Physically, it is understood that the fluid moves fast in a porous medium with more permeability. Figure 5 displays the impression of Hartmann number $M$ over the momentum profile $f(\eta)$. From Figure 5, we noted that as we enhance the Hartmann number the momentum profile declines. Physically, the increase in Hartmann number creates Lorentz force, which provides the opposing force to the flow as a result retardation in the flow. Another very novel physical parameter in the motion of nanofluid is the Reynolds number Re; the impact of this Reynolds number Re on the momentum profile $f(\eta)$ of the couple stress nanofluid is portrayed in Figure 6. From this Figure 6, it is observed that as the Reynolds number Re increases, the momentum profile $f(\eta)$ upsurges.

The impact of local inertial parameter Fr on the momentum profile $g(\eta)$ is portrayed in Figure 7. From the same figure, it is noted that momentum profile $g(\eta)$ declines with escalation of inertial parameter Fr. The impact of couple stress parameter $K$ over the momentum profile $g(\eta)$ is demonstrated in Figure 8. From Figure 8, it is distinguished that the momentum profile $g(\eta)$ declines by the enhancing of couple stress parameter. Figure 9 portrays the relation of porosity parameter $\lambda$ versus momentum profile $g(\eta)$ of the couple stress nanofluid. Figure 9 indicates that there is direct relation of porosity parameter $\lambda$ with the momentum profile $g(\eta)$ of the couple stress nanofluid.

Figure 10 indicates the effect of Brownian parameter $\mathrm{Nb}$ over the concentration profile $\phi(\eta)$ of the couple stress nanofluid past a rotting disc. From Figure 10, it is noted that the concentration profile $\phi(\eta)$ of the couple stress nanofluid declines with the escalation of the Brownian parameter $\mathrm{Nb}$. Figure 11 demonstrates the impact of thermophoresis

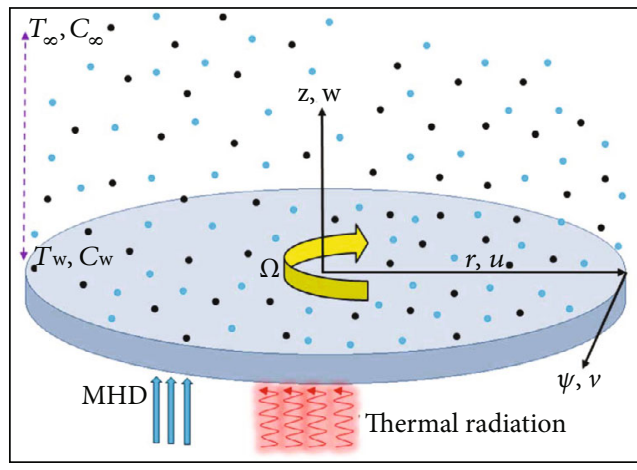

FIgUre 1: Pictorial sketch of the spinning flow.

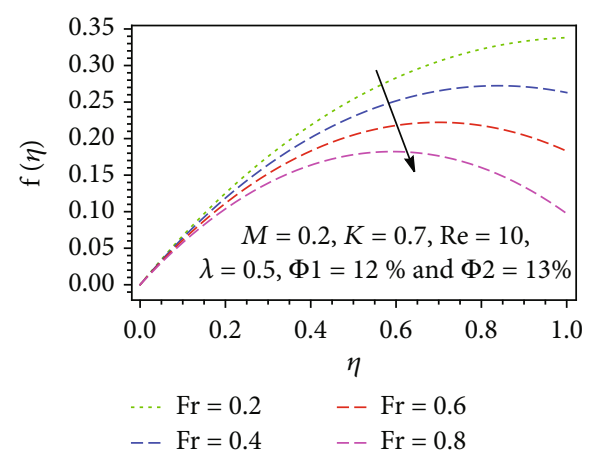

FIgURE 2: Impact of local inertial parameter Fr over momentum profile $f(\eta)$.

parameter Nt over the concentration profile $\phi(\eta)$ of the couple stress nanofluid. From Figure 11, we observed that as the thermophoresis parameter enhances the concentration profile of the nanofluid enhances. From Figure 12, it is noted that the concentration $\phi(\eta)$ of the nanoparticles becomes low where the Reynolds number Re is high during the flow. Figure 13 portrayed the influence of Schmidt number Sc over the concentration profile $\phi(\eta)$ of the nanofluid. From Figure 13, it is obvious that the concentration $\phi(\eta)$ of the couple stress nanofluid declines with the escalation of the Schmidt number Sc.

Figure 14 is the sketch of energy profile $\theta(\eta)$ against local inertial parameter Fr. From Figure 14, it is very clear that the energy profile $\theta(\eta)$ of the couple stress nanofluid enhances as we increase the inertial parameter $\mathrm{Fr}$ of the medium. Physically, the increase in inertial parameter means the decrease in permeability of the medium which reduces the pace of the fluid provides more time to fluid in contact with hot wall which enhances the temperature profile of the couple stress hybrid nanofluid. Figure 15 demonstrates the effect of couple stress parameter $K$ over the energy profile $\theta(\eta)$ of the flowing couple stress nanofluid. It is very clear from Figure 15 that the energy profile $\theta(\eta)$ drops as the couple stress feature of the fluid boosts. Figure 16 is the sketch of energy profile $\theta(\eta)$ of the couple stress nanofluid against the Brownian parameter $\mathrm{Nb}$. Figure 17 demonstrates the effect of thermophoresis parameter Nt over the energy profile $\theta(\eta)$ of the nanofluid. It is obvious from Figure 17 that 


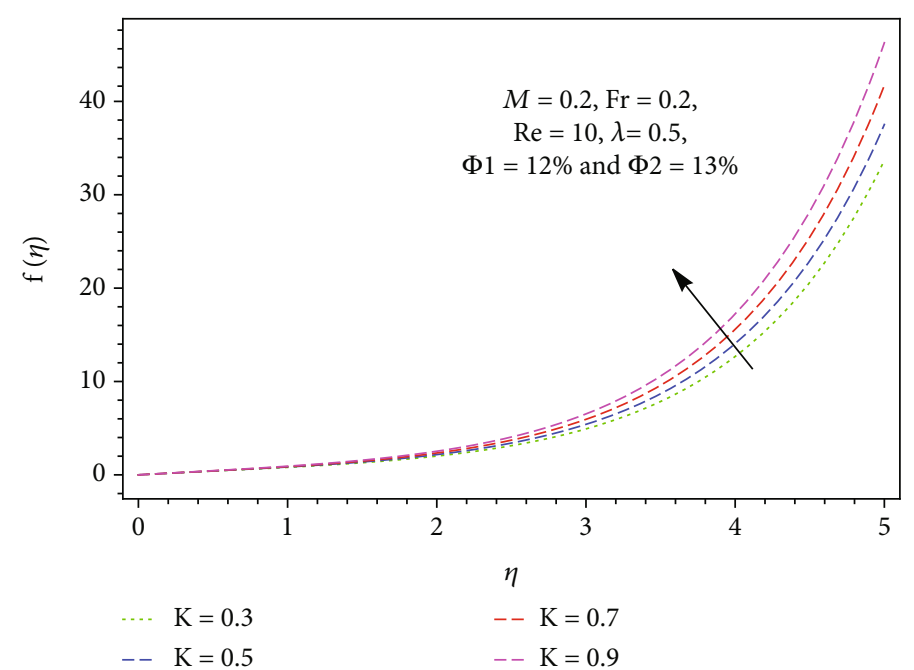

FIGURE 3: Influence of couple stress parameter $K$ over momentum profile $f(\eta)$.

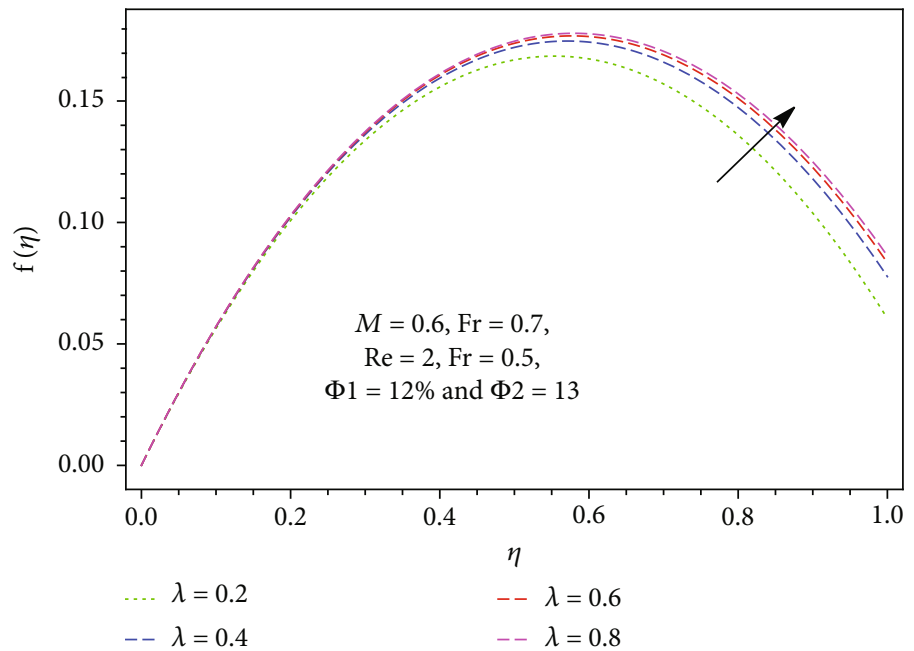

FigURE 4: Influence of porosity parameter $\lambda$ over momentum profile $f(\eta)$.

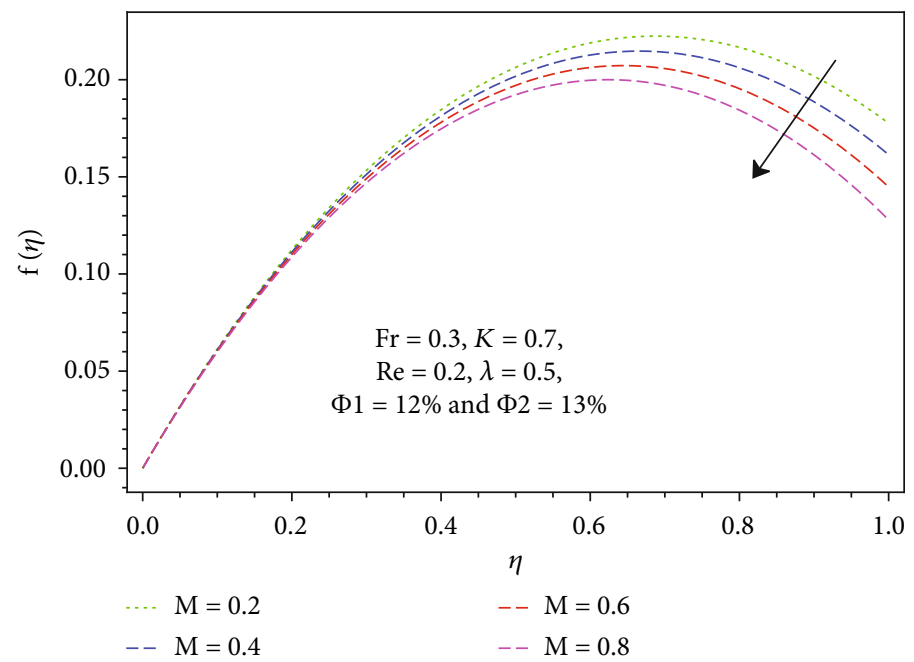

Figure 5: Influence of Hartmann number $M$ over momentum profile $f(\eta)$. 


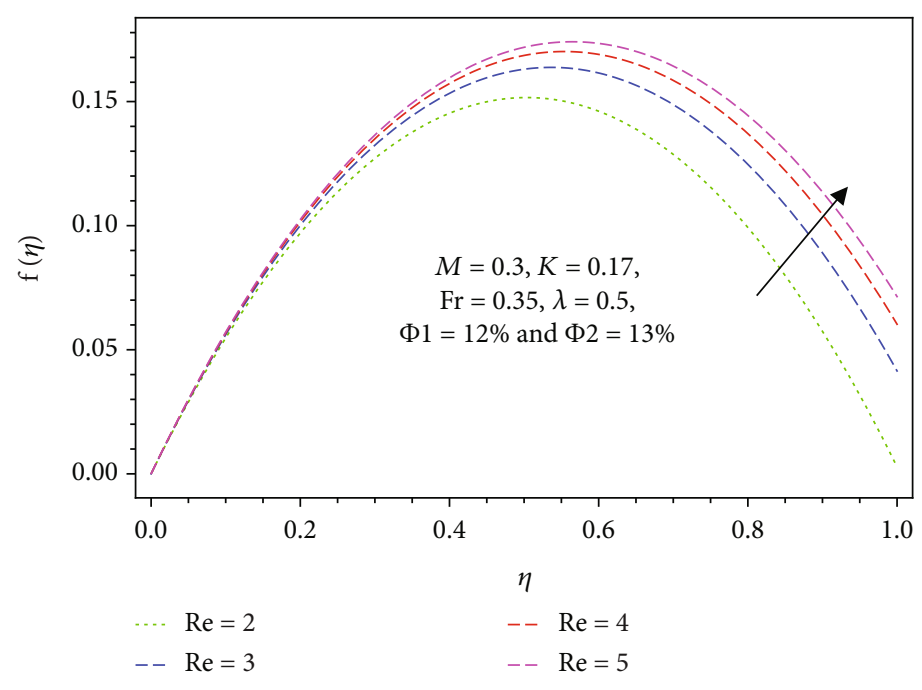

FiguRE 6: Impact of Reynolds number Re over momentum profile $f(\eta)$.

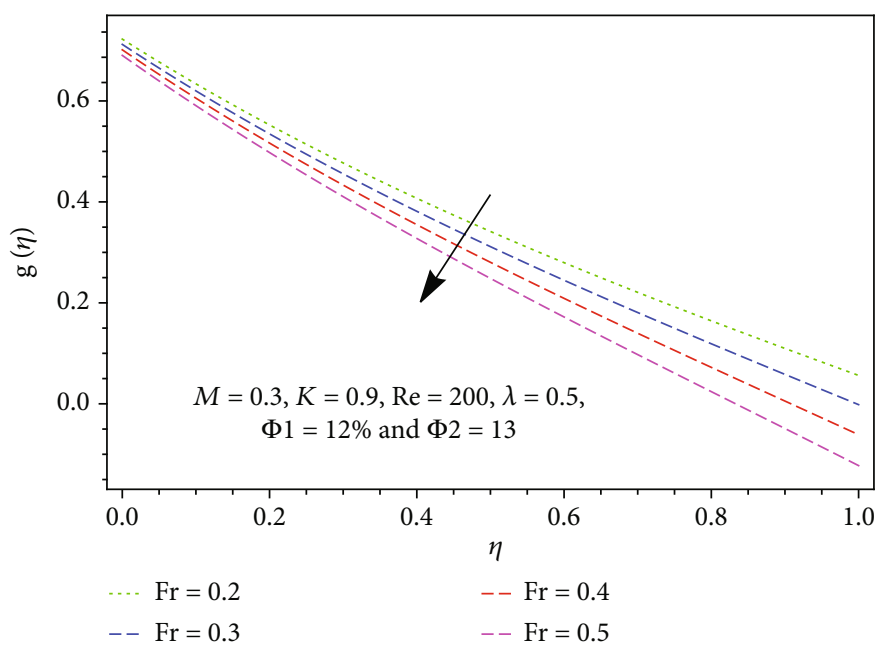

FiguRE 7: Impact of local inertial parameter Fr over momentum profile $g(\eta)$.

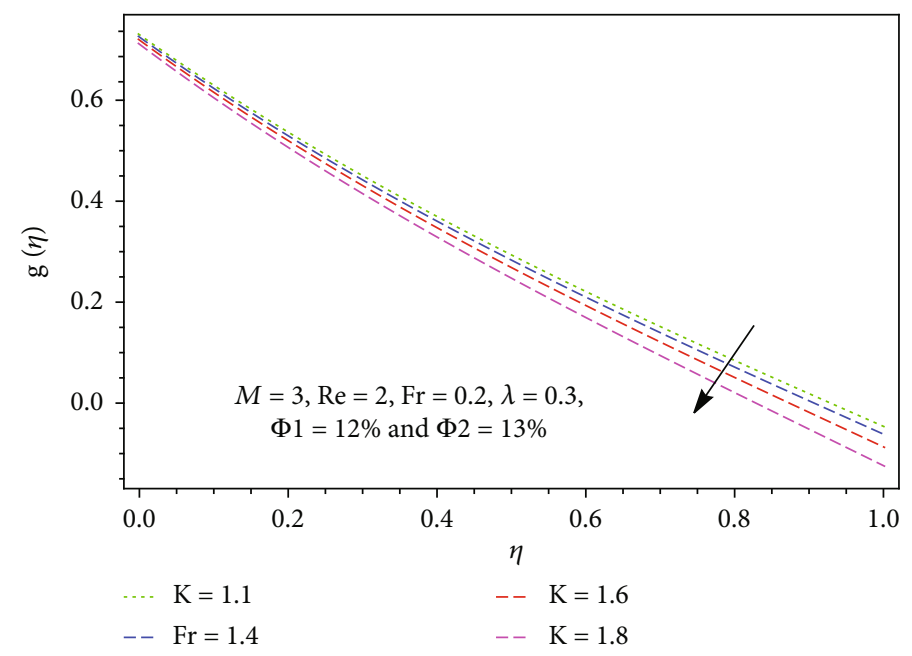

FigurE 8: Impact of couple stress parameter $K$ over momentum profile $g(\eta)$. 


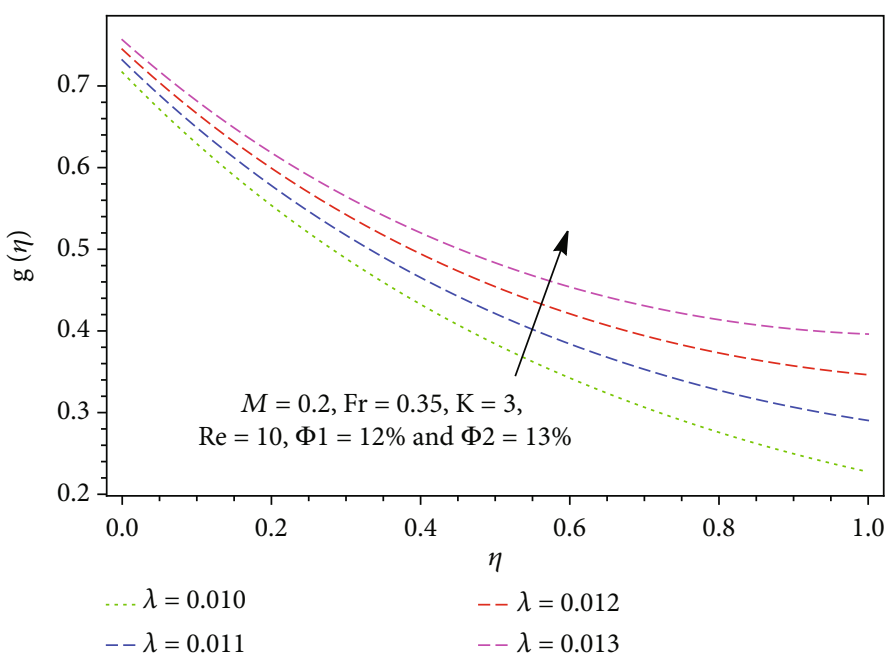

FIGURE 9: Impact of porosity parameter $\lambda$ over momentum profile $g(\eta)$.

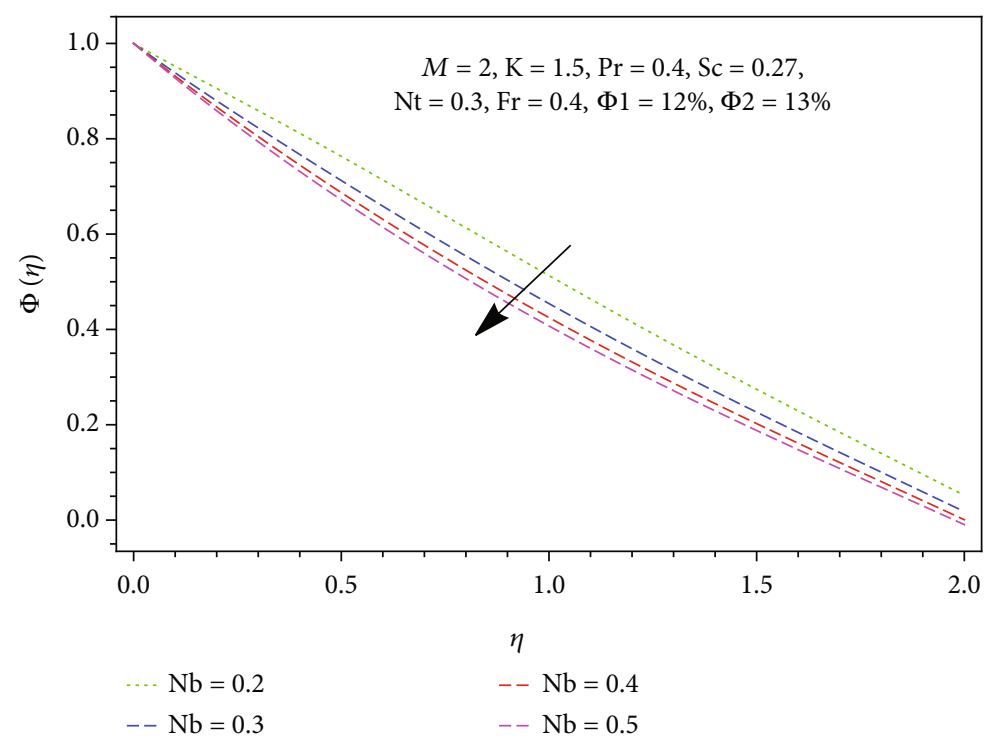

FiguRe 10: Impact of Brownian motion parameter $\mathrm{Nb}$ over concentration profile $\phi(\eta)$.

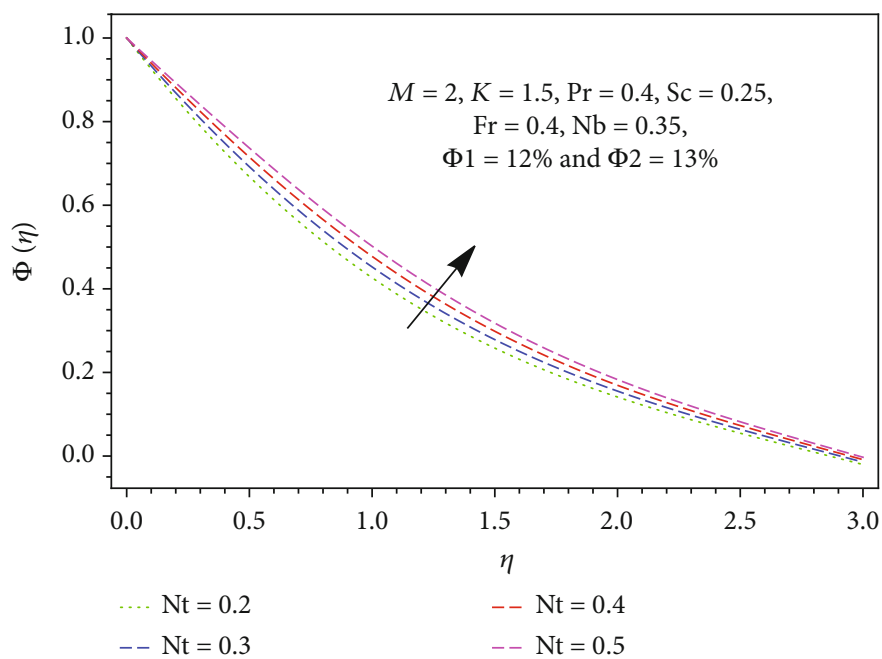

FIGURE 11: Impact of thermophoresis parameter Nt on the concentration profile $f(\eta)$. 


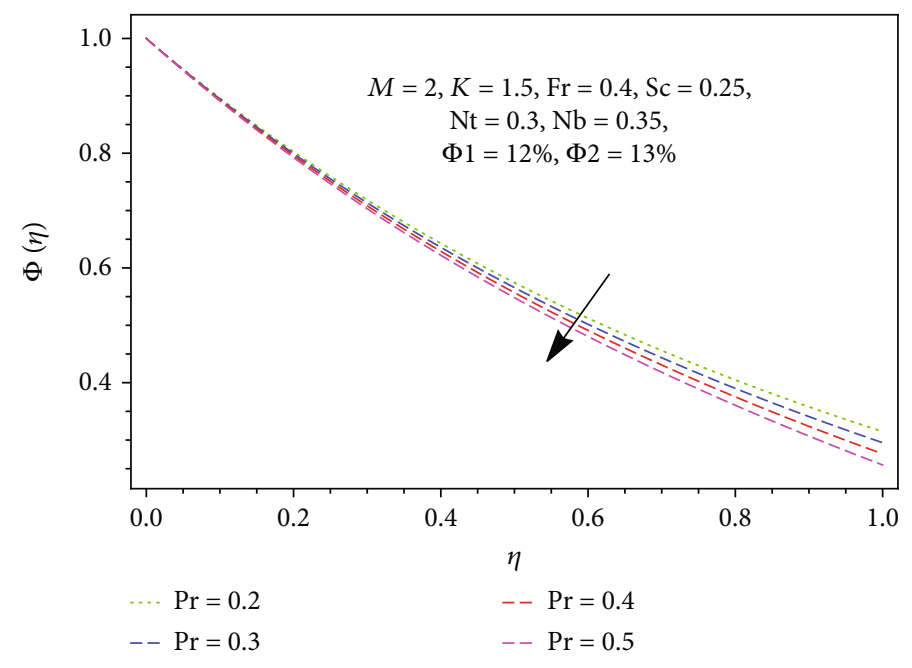

Figure 12: Impact of Prandtl number Pr on the concentration profile $\phi(\eta)$.

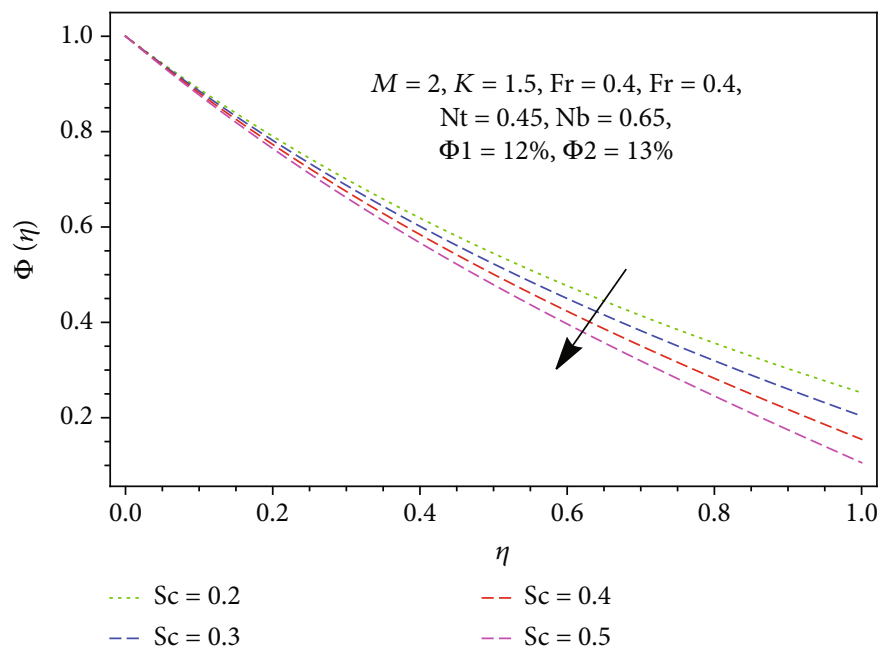

FIGURE 13: Impact of Schmidt number Sc on the concentration profile $\phi(\eta)$.

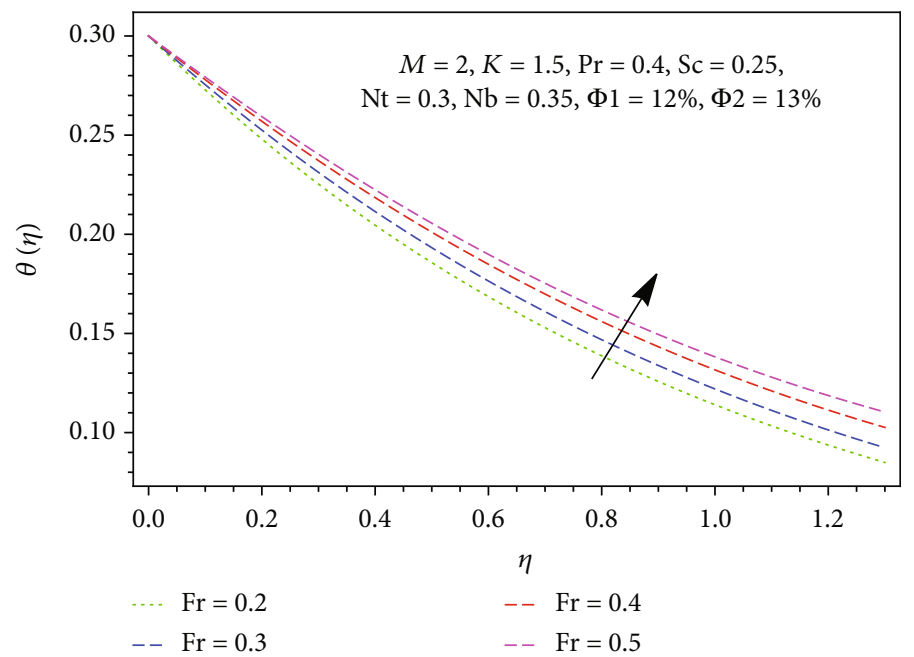

FIGURE 14: Influence of local inertial parameter Fr over energy profile $\theta(\eta)$. 


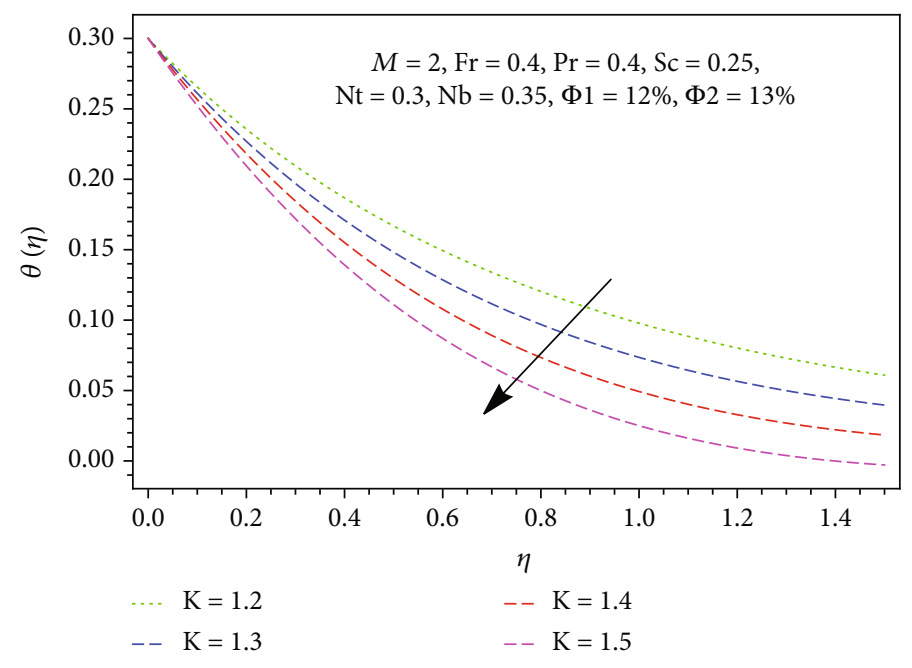

FIGURE 15: Impact of couple stress parameter $K$ on the energy profile $\theta(\eta)$.

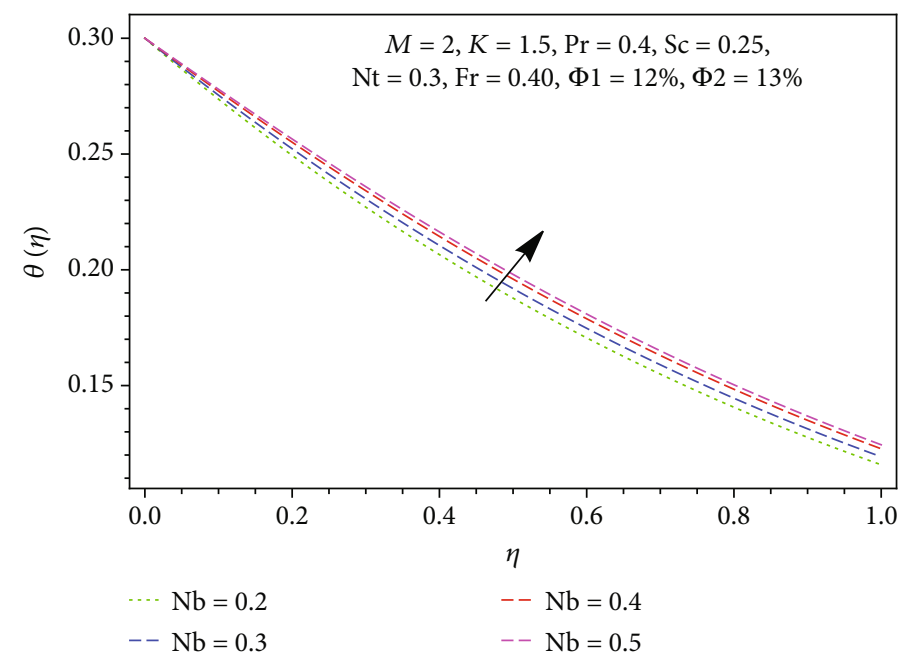

FIGURE 16: Impact of Brownian motion parameter $\mathrm{Nb}$ over energy profile $\theta(\eta)$.

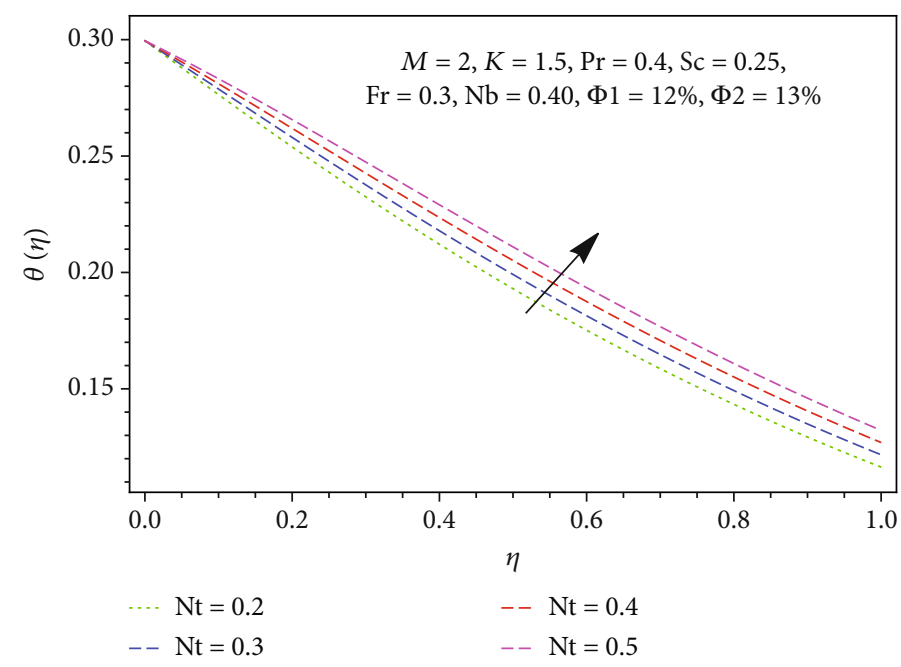

FIGURE 17: Impact of thermophoresis parameter Nt over energy profile $\theta(\eta)$. 


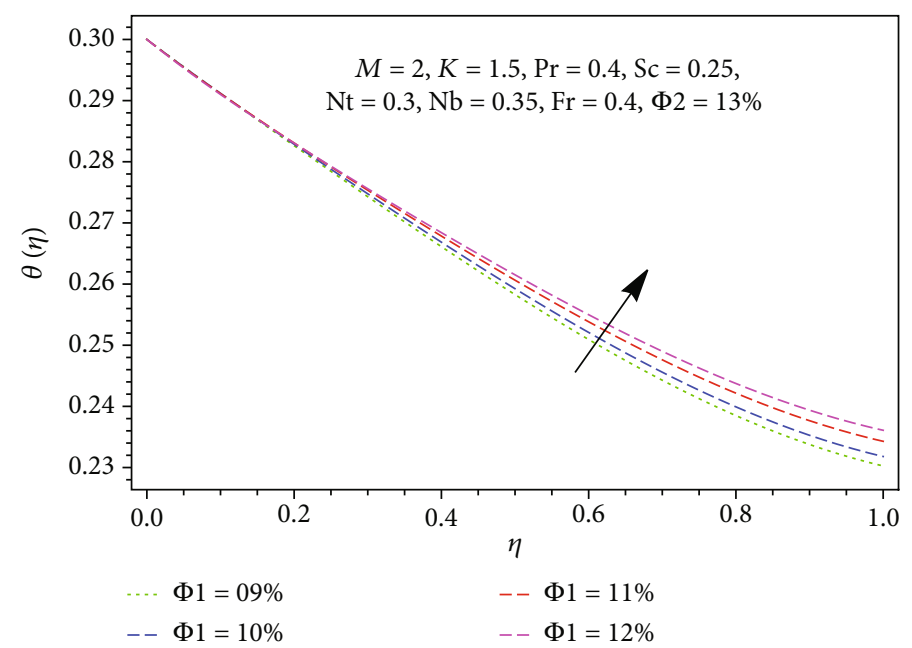

Figure 18: Impact of nanoparticle fraction $\phi_{1}$ over energy profile $\theta(\eta)$.

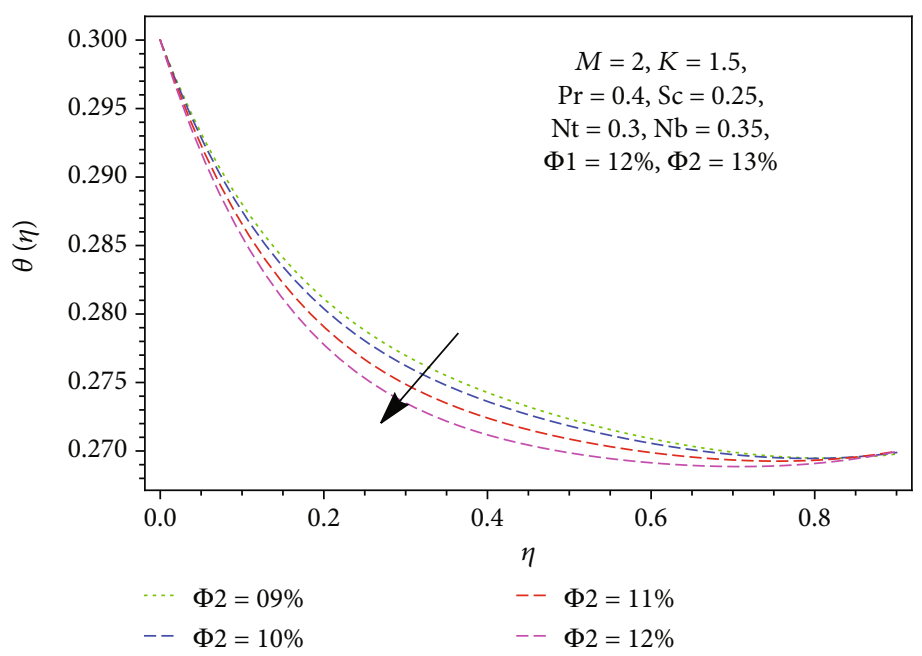

FIgURE 19: Influence of nanoparticle fraction $\phi_{2}$ over energy profile $\theta(\eta)$.

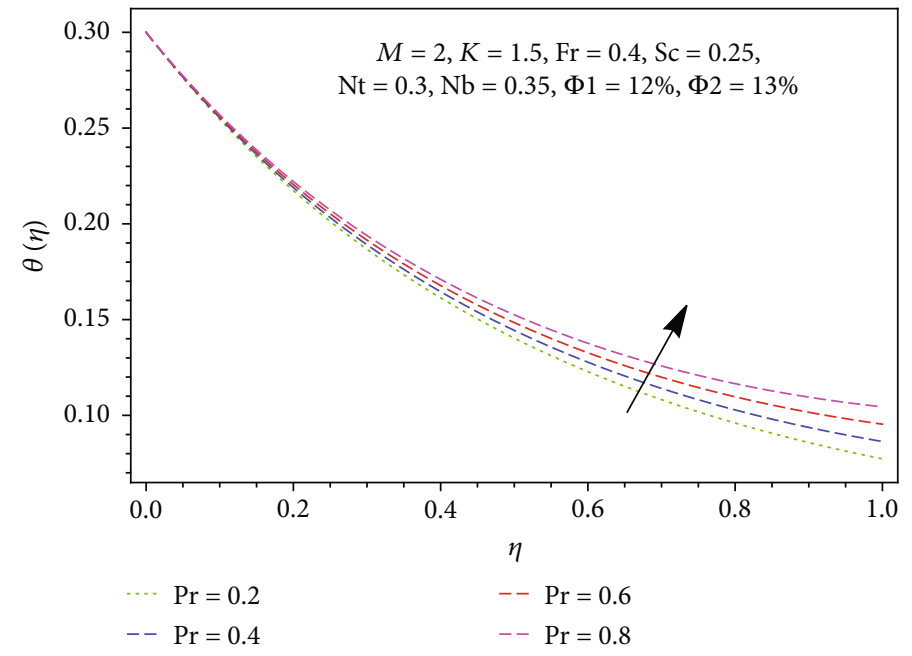

Figure 20: Effect of Prandtl number Pr over momentum profile $f(\eta)$. 


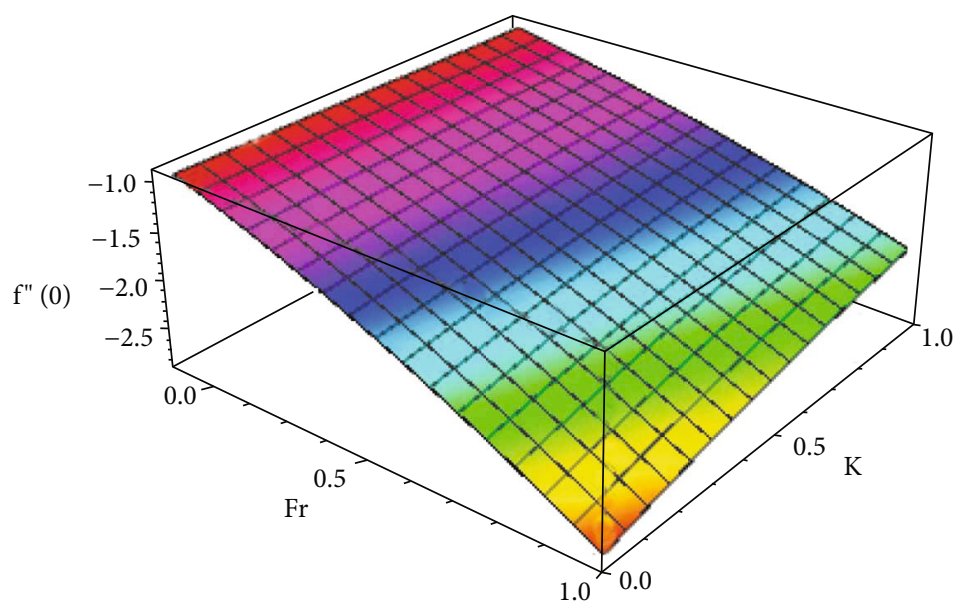

FIgURE 21: Effect of local inertia parameter Fr and couple stress parameter $K$ on skin friction $f^{\prime \prime}(0)$.

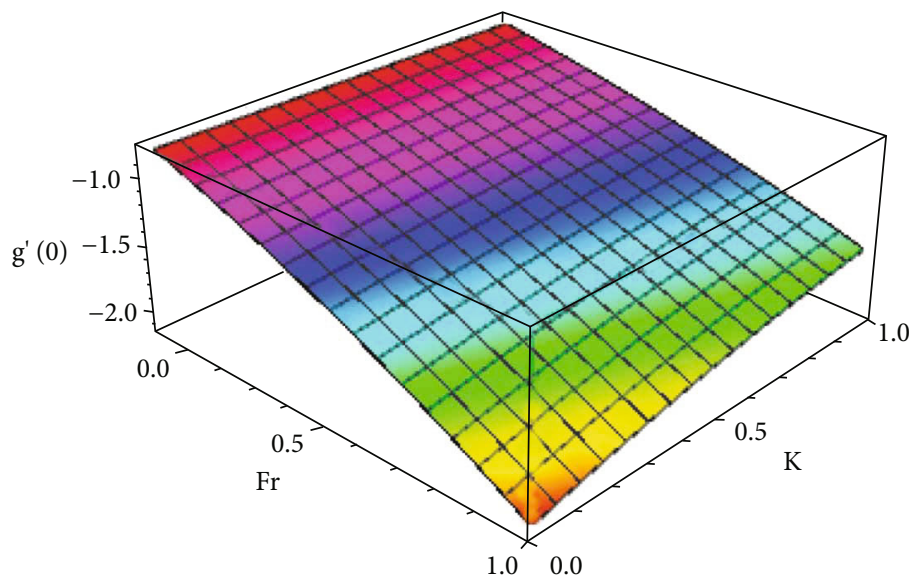

FIGURE 22: Impact of local inertia parameter Fr and couple stress parameter $K$ on skin friction $g^{\prime}(0)$.

TABLE 1: Thermophysical properties of $\mathrm{H}_{2} \mathrm{O}$, titanium oxide, and aluminum oxide.

\begin{tabular}{lccc}
\hline Physical properties & Base fluid & \multicolumn{2}{c}{ Solid nanoparticles } \\
\hline Density $\rho\left(\mathrm{kg} / \mathrm{m}^{3}\right)$ & $\mathrm{H}_{2} \mathrm{O}$ & $\mathrm{TiO}_{2}$ & 4230 \\
Thermal conductivity $\kappa(\mathrm{W} / \mathrm{mK})$ & 997.1 & 8.4 & 4000 \\
Specific heat capacity $c_{p}(\mathrm{~J} / \mathrm{kgK})$ & 0.613 & 692 & 40 \\
Electric conductivity $\sigma\left(\mathrm{Sm}^{-1}\right)$ & 4179 & $2.38 \times 10^{6}$ & $5.96 \times 10^{7}$ \\
\hline
\end{tabular}

the energy profile $\theta(\eta)$ boosts as the thermophoresis parameter Nt upsurges. Figure 18 is the plot of energy profile $\theta(\eta)$ of the flowing fluid against the volume fraction $\phi_{1}$ of the titanium oxide $\mathrm{TiO}_{2}$. From Figure 18, it is very clear that the energy profile $\theta(\eta)$ enhances with the escalation of nanoparticles volume fraction $\phi_{1}(9 \%, 10 \%, 11 \%$, and $12 \%)$ of the titanium oxide $\mathrm{TiO}_{2}$ in the base fluid $\left(\mathrm{H}_{2} \mathrm{O}\right)$. Figure 19 indicates that as we increase the volume fraction $\phi_{2}$ $(9 \%, 10 \%, 11 \%$, and $12 \%)$ of the nanoparticles, aluminum oxide $\mathrm{Al}_{2} \mathrm{O}_{3}$ enhancing the energy profile $\theta(\eta)$ of the couple stress nanofluid declines. Figure 20 indicates the relation of energy profile $\theta(\eta)$ with the Prandtl number Pr. From Figure 20, it is noted that the energy profile enhances as the Prandtl number Pr enhances.

Figures 21 and 22 show the combine impact of local inertial parameter $\mathrm{Fr}$ and couple stress parameter $K$ over the skin friction coefficient $\operatorname{Re}^{1 / 2} C_{f}$ and $\operatorname{Re}^{1 / 2} C_{g}$. From Figures 21 and 22, we see clearly that with the increase in inertial parameter Fr the decrease in permeability increase the pressure drop due to this the velocity of the nanofluid decreases this generates the reduction in the skin fraction $\operatorname{Re}^{1 / 2} C_{g}$. Also, we see from Figures 21 and 22 that with the 
TABLE 2: Impact of some physical parameters over skin friction coefficient $\operatorname{Re}^{1 / 2} C_{f}$ and $\operatorname{Re}^{1 / 2} C_{g}$ when $R=0.3, \theta_{w}=0.4, s_{t}=0.7, \gamma^{*}$ $=0.3$, and $\gamma=0.3$ when $\phi_{1}=12 \%$ and $\phi_{2}=13 \%$.

\begin{tabular}{ccccccc}
\hline Fr & $M$ & $K$ & $\mathrm{Re}$ & $\lambda$ & $\mathrm{Re}^{1 / 2} C_{f}$ & $\mathrm{Re}^{1 / 2} C_{g}$ \\
\hline 0.5 & 0.45 & 0.35 & 0.25 & 0.5 & 0.60245520 & 0.7633975 \\
0.6 & & & & & 0.3533196 & 0.4935312 \\
0.7 & & & & & 0.08601030 & 0.2042981 \\
& 0.5 & & & & 0.0835144 & 0.2017363 \\
& 0.6 & & & & 0.0785259 & 0.1966159 \\
& 0.7 & & & & 0.0735418 & 0.1914999 \\
& & 0.5 & & & 0.2390841 & 0.3674829 \\
& 0.6 & & & 0.3472050 & 0.4823447 \\
& 0.7 & & & 0.4601849 & 0.6020653 \\
& & 0.5 & & 0.1058902 & 0.2247019 \\
& & 0.6 & & 0.1092103 & 0.2281093 \\
& & 0.7 & & 0.1115829 & 0.2305443 \\
& & & 0.5 & 0.0860103 & 0.2042981 \\
& & & 0.6 & 0.0926293 & 0.2110917 \\
& & & 0.7 & 0.0973618 & 0.2159489 \\
\hline
\end{tabular}

TABLE 3: Impact of various physical parameters over the Nusselt number $(1 / 2) \operatorname{Re}^{1 / 2} \mathrm{Nu}$ when $R=0.3, \gamma^{*}=0.3$, and $\gamma=0.3$ when $\phi_{1}$ $=12 \%$ and $\phi_{2}=13 \%$.

\begin{tabular}{cccccc}
\hline$R d$ & $\theta_{w}$ & $s_{t}$ & $\mathrm{Nt}$ & $\mathrm{Nb}$ & $1 / 2 \mathrm{Re}^{1 / 2} \mathrm{Nu}$ \\
\hline 0.4 & 0.52 & 0.34 & 0.23 & 0.27 & 0.4354549 \\
0.5 & & & & & 0.5151867 \\
0.6 & & & & & 0.5949184 \\
& 0.4 & & & & 0.5263548 \\
& 0.5 & & & & 0.4478600 \\
& 0.6 & & & & 0.3936489 \\
& 0.4 & & & 0.4030697 \\
& 0.5 & & & 0.3613787 \\
& 0.6 & & & 0.3303248 \\
& & 0.4 & & 0.4291929 \\
& & 0.5 & & 0.4255094 \\
& & 0.6 & & 0.4218259 \\
& & & 0.4 & 0.4194930 \\
& & & 0.5 & 0.4072145 \\
& & & 0.6 & 0.3949361 \\
\hline & & & &
\end{tabular}

increase in couple stress parameter the velocity field enhances due to which the skin fraction $\operatorname{Re}^{1 / 2} C_{g}$ enhances. Table 1 shows the thermophysical properties of the base fluid (water $\mathrm{H}_{2} \mathrm{O}$ ) and solid nanoparticles $\left(\mathrm{TiO}_{2}, \mathrm{Al}_{2} \mathrm{O}_{3}\right.$ ). Also, Table 2 displays the impact of various physical parameters over the skin friction coefficient when the nanoparticle volume friction in the base fluid is $\phi_{1}=12 \%\left(\mathrm{TiO}_{2}\right)$ and $\phi_{2}$
$=13 \%\left(\mathrm{Al}_{2} \mathrm{O}_{3}\right)$. Table 3 displays the impact of various physical parameters over the heat transfer rate through the couple stress hybrid nanofluid when there is $12 \%$ volume fraction of the titanium oxide $\left(\mathrm{TiO}_{2}\right)$ nanoparticles and $13 \%$ volume fraction of the aluminum oxide $\left(\mathrm{Al}_{2} \mathrm{O}_{3}\right)$ nanoparticles in $75 \%$ water $\left(\mathrm{H}_{2} \mathrm{O}\right)$.

\section{Main Findings}

(i) Velocity $f(\eta)$ enhances with the increase in local inertial parameter Fr, couple stress parameter $K$, porosity parameter $\lambda$, and Reynolds number $\mathrm{Re}$ but declines by the escalation in Hartmann number $M$. Also, the velocity $g(\eta)$ decreases with the increase in local inertial parameter Fr and porosity parameter $\lambda$ but decreases with an escalation in couple stress parameter $K$

(ii) Concentration profile $\phi(\eta)$ decreases with the increase in $\mathrm{Nb}, \mathrm{Pr}$, and $\mathrm{Sc}$ but enhances with $\mathrm{Nt}$

(iii) Temperature profile $\theta(\eta)$ decreases with the increase inFr, $\phi_{2}$, and $K$ but enhances with $\mathrm{Nt}, \mathrm{Nb}$ , $\phi_{1}$, and $\operatorname{Pr}$

(iv) Skin friction enhances with the increase in local inertial parameter Fr, couple stress parameter $K$, Reynolds number $\mathrm{Re}$, and porosity parameter $\lambda$ but decreases for Hartmann number $M$. Also, the heat transfer rate $(1 / 2) \mathrm{Re}^{1 / 2} \mathrm{Nu}$ enhances for radiation parameter $R d$ but decreases in $\theta_{w}$ (variable for temperature), $s_{t}$ (the thermal stratification parameter), thermophoresis parameter $\mathrm{Nt}$, and Brownian parameter $\mathrm{Nb}$

\section{Abbreviations}

RK: Runge-Kutta

TD: Tiwari and Das

MG: Maxwell-Garnett

HAM: Homotopy analysis method

PDEs: Partial differential equations

ODEs: Ordinary differential equation

MRT: Multirelaxation time

LBM: Lattice Boltzmann method.

\section{Nomenclature}

$u, v, w: \quad$ Velocity components (m)

$(r, \psi, z)$ : Coordinate direction

$\varepsilon(z): \quad$ Variable porosity function

$K(z)$ : Variable permeability function

$\sigma: \quad$ Electric conductivity $(\mathrm{S} / \mathrm{m})$

$\sigma^{*}: \quad$ Stefan-Boltzmann constant $\left(\mathrm{Wm}^{-2} \mathrm{~K}^{-4}\right)$

$\beta_{0}$ : $\quad$ Magnetic field (Tesla)

$\rho: \quad$ Density $\left(\mathrm{kg} / \mathrm{m}^{3}\right)$

$\eta_{0}$ : Material constant for couple stress parameter

$C_{b}: \quad$ Drag coefficient

$k: \quad$ Mean absorption coefficient

$D_{B}$ : $\quad$ Brownian diffusion coefficient $\left(\mathrm{m}^{2} / \mathrm{s}\right)$ 
$D_{T}: \quad$ Thermophoresis diffusion coefficient

$\tau: \quad$ Ratio between heat capacity of solid particles to heat capacity of base fluid

$\Omega: \quad$ System angular velocity ( $\mathrm{rev} / \mathrm{min})$

$L: \quad$ Velocity slip coefficient

$\varepsilon_{\infty}: \quad$ Porosity proportionality constant

$K_{\infty}: \quad$ Permeability proportionality constant

$T_{w}$ : $\quad$ Thermal stratification at the disc (K)

$T_{\infty}$ : Thermal stratification at the free stream $(\mathrm{K})$

$T_{0}$ : $\quad$ Reference temperature $(\mathrm{K})$

$\lambda$ : $\quad$ Porosity parameter

Re: $\quad$ Reynolds number

$d: \quad$ Variable permeability

$d^{*}: \quad$ Variable porosity

Fr: $\quad$ Local inertial parameter

M: $\quad$ Hartman number

$K: \quad$ Couple stress parameter

$R: \quad$ Radiation parameter

Pr: $\quad$ Prandtl number

Nt: Thermophoresis parameter

$\mathrm{Nb}$ : Brownian motion parameter

Sc: $\quad$ Schmidt number

$\gamma$ : $\quad$ Parameter

$\kappa: \quad$ Thermal conductivity $\left(\mathrm{Wm}^{-1} \mathrm{~K}^{-1}\right)$

$c_{p}: \quad$ Specific heat capacity $(\mathrm{J} / \mathrm{K})$

$\eta_{0}: \quad$ Material constant for couple stress parameter

$\alpha_{\text {hnf }}: \quad$ Thermal diffusivity of hybrid nanofluid

$\theta_{w}: \quad$ Variable for temperature

$s_{t}: \quad$ Thermal stratification parameter

\section{Subscript}

hnf: Hybrid nanofluid

$f$ : $\quad$ Base fluid

0: $\quad$ Reference point

1: $\quad$ Related to first solid nanoparticles

2: $\quad$ Related to second solid nanoparticles

$w$ : At the wall level

$\infty$ : At the free stream level.

\section{Data Availability}

Complete data available inside the manuscript.

\section{Conflicts of Interest}

The authors declare that they have no competing interest.

\section{References}

[1] S. U. Choi and J. A. Eastman, Enhancing Thermal Conductivity of Fluids with Nanoparticles, vol. 231, no. 1995, 1995, Argonne National Lab, IL, United States, 1995.

[2] A. T. Akinshilo and G. M. Sobamowo, "Perturbation solutions for the study of MHD blood as a third grade nanofluid transporting gold nanoparticles through a porous channel," Journal of Applied and Computational Mechanics, vol. 3, no. 2, pp. 103-117, 2017.

[3] D. Madhesh, R. Parameshwaran, and S. Kalaiselvam, "Experimental investigation on convective heat transfer and rheolog- ical characteristics of $\mathrm{Cu}-\mathrm{TiO}_{2}$ hybrid nanofluids," Experimental Thermal and Fluid Science, vol. 52, pp. 104115, 2014.

[4] S. Senthilaraja, K. Vijayakumar, and R. Ganadevi, "A comparative study on thermal conductivity of $\mathrm{Al}_{2} \mathrm{O}_{3}$ /water, $\mathrm{CuO}$ /water and $\mathrm{Al}_{2} \mathrm{O}_{3}-\mathrm{CuO}$ /water nanofluids," Digest Journal of Nanomaterials and Biostructures, vol. 10, pp. 14491458, 2015.

[5] G. H. Bhosale and S. L. Borse, "Pool boiling CHF enhancement with $\mathrm{Al}_{2} \mathrm{O}_{3}-\mathrm{CuO} / \mathrm{H}_{2} \mathrm{O}$ hybrid nanofluid," International Journal of Engineering Research \& Technology, vol. 2, no. 10, pp. 946-950, 2013.

[6] Y. He, S. Vasiraju, and L. Que, "Hybrid nanomaterial-based nanofluids for micropower generation," RSC Advances, vol. 4, no. 5, pp. 2433-2439, 2014.

[7] M. H. Esfe, A. A. Abbasian Arani, M. Rezaie, W.-M. Yan, and A. Karimipour, "Experimental determination of thermal conductivity and dynamic viscosity of $\mathrm{Ag}-\mathrm{MgO} /$ water hybrid nanofluid," International Communications in Heat and Mass Transfer, vol. 66, pp. 189-195, 2015.

[8] L. Syam Sundar, G. O. Irurueta, R. E. Venkata, M. K. Singh, and A. C. M. Sousa, "Thermal conductivity and viscosity of hybrid nanfluids prepared with magnetic nanodiamondcobalt oxide (ND- $\mathrm{Co}_{3} \mathrm{O}_{4}$ ) nanocomposite," Thermal Engineering, vol. 7, pp. 66-77, 2016.

[9] A. A. Minea, A Review on the Thermophysical Properties of Water-Based Nanofluids and their Hybrids, the Annals of "DUNAREA DE JOS", University of GALATI, 2016.

[10] F. Haidera, T. Hayat, and A. A. Saedi, "Flow of hybrid nanofluid through Darcy-Forchheimer porous space with variable characteristics," Alexandria Engineering Journal, vol. 60, no. 3, pp. 3047-3056, 2021.

[11] N. Acharya, R. Bag, and P. K. Kundu, "Influence of Hall current on radiative nanofluid flow over a spinning disk: a hybrid approach," Physica E: Low-dimensional Systems and Nanostructures, vol. 111, pp. 103-112, 2019.

[12] U. Farooq, M. I. Afridi, M. Qasim, and D. C. Lu, "Transpiration and viscous dissipation effects on entropy generation in hybrid nanofluid flow over a nonlinear radially stretching disk," Entropy, vol. 20, p. 668, 2018.

[13] S. Islam, A. Khan, P. Kumam et al., "Radiative mixed convection flow of maxwell nanofluid over a stretching cylinder with joule heating and heat source/sink effects," Scientific Reports, vol. 10, pp. 1-18, 2020.

[14] M. Zubair, Z. Shah, S. Islam, W. Khan, and A. Dawar, "Study of Three dimensional Darcy-Forchheimer squeezing nanofluid flow with Cattaneo-Christov heat flux based on four different types of nanoparticles through entropy generation analysis," Advances in Mechanical Engineering, vol. 11, no. 5, p. 17, 2019.

[15] S. Khan, S. A. Shehzad, A. Rauf, and N. Ali, "Mixed convection flow of couple stress nanofluid over oscillatory stretching sheet with heat absorption/generation effects," Results in Physics, vol. 8, pp. 1223-1231, 2018.

[16] A. Khadeeja and A. Asim, "Transport and heat transfer of time dependent MHD slip flow of nanofluids in solar collectors with variable thermal conductivity and thermal radiation," Results in Physics, vol. 6, pp. 746-753, 2016.

[17] T. Hayat, M. B. Ashraf, S. A. Shehzad, and N. N. Bayomi, "Mixed convection flow of viscoelastic nanofluid over a stretching cylinder," Journal of the Brazilian Society of 
Mechanical Sciences and Engineering, vol. 37, no. 3, pp. 849859, 2015.

[18] M. Turkyilmazoglu, "Unsteady convection flow of some nanofluids past a moving vertical flat plate with heat transfer," Journal of heat transfer, vol. 136, article 031704, 2014.

[19] N. A. Haroun, P. Sibanda, S. Mondal, and S. S. Motsa, "On unsteady MHD mixed convection in a nanofluid due to a stretching/shrinking surface with suction/injection using the spectral relaxation method," Boundary value problems, vol. 2015, no. 1, p. 17, 2015.

[20] S. Mirmasoumi and A. Behzadmehr, "Numerical study of laminar mixed convection of a nanofluid in a horizontal tube using two-phase mixture model," Applied Thermal Engineering, vol. 28, no. 7, pp. 717-727, 2008.

[21] A. Mahdy, "Unsteady mixed convection boundary layer flow and heat transfer of nanofluids due to stretching sheet," Nuclear Engineering and Design, vol. 249, no. 248, pp. 248255, 2012.

[22] G. Vasumathi and J. A. Rao, "Brownian motion and thermophoresis effects on mixed convection boundary layer flow of nanofluid over an exponentially stretching sheet with Joule heating," Journal of Nanofluids, vol. 4, no. 4, pp. 505-511, 2015.

[23] C. Sumalatha and B. Shanker, "MHD mixed convection flow of a nanofluid over a nonlinear stretching sheet with variable wall temperature and concentration," Journal of Nanofluids, vol. 5, no. 5, pp. 707-712, 2016.

[24] J. P. Kumar, J. C. Umavathi, B. J. Gireesha, and M. K. Prasad, "Mixed convective flow in a vertical double passage channel filled with nanofluid using Robin boundary conditions," Journal of Nanofluids, vol. 5, no. 4, pp. 549-559, 2016.

[25] S. A. Shehzad, F. M. Abbasi, T. Hayat, and F. Alsaadi, "MHD mixed convective peristaltic motion of nanofluid with Joule heating and thermophoresis effects," PLoS One, vol. 9, no. 11, article e0111417, 2014.

[26] A. Malvandi, M. R. Safaei, M. H. Kaffash, and D. D. Ganji, "MHD mixed convection in a vertical annulus filled with $\mathrm{Al}_{2} \mathrm{O}_{3}$-water nanofluid considering nanoparticle migration," Journal of Magnetism and Magnetic Materials, vol. 382, pp. 296-306, 2015.

[27] F. Selimefendigil, H. F. Öztop, and N. Abu-Hamdeh, "Mixed convection due to rotating cylinder in an internally heated and flexible walled cavity filled with $\mathrm{SiO}_{2}$-water nanofluids: effect of nanoparticle shape," International Communications in Heat and Mass Transfer, vol. 71, pp. 9-19, 2016.

[28] T. Hayat and S. Nadeem, "Heat transfer enhancement with Ag-CuO/water hybrid nanofluid," Results in Phyics, vol. 7, pp. 2317-2324, 2017.

[29] A. M. Rashad, A. J. Chamkha, M. A. Ismael, and T. Salah, "Magnetohydrodynamics natural convection in a triangular cavity filled with a $\mathrm{Cu}-\mathrm{Al}_{2} \mathrm{O}_{3}$ /water hybrid nanofluid with localized heating from below and internal heat generation," Journal of Heat Transfer, vol. 140, no. 7, article 072502, 2018.

[30] M. Usman, M. Hamid, T. Zubair, R. U. Haq, and W. Wang, "_Cu- $\mathrm{Al}_{2} \mathrm{O}_{3-}$ / water hybrid nanofluid through a permeable surface in the presence of nonlinear radiation and variable thermal conductivity via LSM," International Journal of Heat and Mass Transfer, vol. 126, pp. 1347-1356, 2018.

[31] M. A. Mansour, S. Siddiqa, R. S. R. Gorla, and A. M. Rashad, "Effects of heat source and sink on entropy generation and MHD natural convection of $\mathrm{Al}_{2} \mathrm{O}_{3}$ - $\mathrm{Cu}$ /water hybrid nanofluid filled with square porous cavity," Thermal Science and Engineering Progress, vol. 6, pp. 57-71, 2018.

[32] M. Sheikholeslami, S. A. M. Mehryan, A. Shafee, and M. A. Sheremet, "Variable magnetic forces impact on magnetizable hybrid nanofluid heat transfer through a circular cavity," Journal of Molecular Liquids, vol. 277, pp. 388-396, 2019.

[33] V. Kumar and J. Sarkar, "Numerical and experimental investigations on heat transfer and pressure drop characteristics of $\mathrm{Al}_{2} \mathrm{O}_{3}-\mathrm{TiO}_{2}$ hybrid nanofluid in minichannel heat sink with different mixture ratio," Powder Technology, vol. 345, pp. 717-727, 2019.

[34] Z. Boulahia, C. Boulahia, and R. Sehaqui, "Two-phase computation of free convection and entropy generation inside an enclosure filled by a hybrid $\mathrm{Al}_{2} \mathrm{O}_{3}-\mathrm{TiO}_{2}$ - $\mathrm{Cu}$ water nanofluid having a corrugated heat source using the generalized Buongiorno's mathematical model: employment of finite volume method," Materials Today, vol. 30, pp. 1056-1067, 2020.

[35] W. T. Urmi, M. M. Rahman, and W. A. W. Hamzah, “An experimental investigation on the thermophysical properties of $40 \%$ ethylene glycol based $\mathrm{TiO}_{2}-\mathrm{Al}_{2} \mathrm{O}_{3}$ hybrid nanofluids," International Communications in Heat and Mass Transfer, vol. 116, article 104663, 2020.

[36] P. Forchheimer, "Wasserbewegung durch boden," CiNii, vol. 45, pp. 1782-1788, 1901.

[37] M. Muskat, The Flow of Homogeneous Fluids through Porous Media, 1946.

[38] M. A. Seddeek, "Influence of viscous dissipation and thermophoresis on Darcy-Forchheimer mixed convection in a fluid saturated porous media," Journal of Colloid and Interface Science, vol. 293, no. 1, pp. 137-142, 2006.

[39] M. A. Sadiq and T. Hayat, "Darcy-Forchheimer flow of magneto Maxwell liquid bounded by convectively heated sheet," Results in Physics, vol. 6, pp. 884-890, 2016.

[40] S. A. Bakar, N. M. Arifin, R. Nazar, F. M. Ali, and I. Pop, "Forced convection boundary layer stagnation-point flow in Darcy-Forchheimer porous medium past a shrinking sheet," Frontiers Heat Mass Transfer, vol. 7, p. 38, 2016.

[41] J. C. Umavathi, O. Ojjela, and K. Vajravelu, "Numerical analysis of natural convective flow and heat transfer of nanofluids in a vertical rectangular duct using Darcy-ForchheimerBrinkman model," International Journal of Thermal Sciences, vol. 111, pp. 511-524, 2017.

[42] T. Hayat, F. Haider, T. Muhammad, and A. Alsaedi, "On Darcy-Forchheimer flow of carbon nanotubes due to a rotating disk," International Journal of Heat and Mass Transfer, vol. 112, pp. 248-254, 2017.

[43] Z. Shah, A. Dawar, S. Islam, I. Khan, and D. L. C. Ching, "Darcy-Forchheimer flow of radiative carbon nanotubes with microstructure and inertial characteristics in the rotating frame," Thermal Engineering, vol. 12, pp. 823-832, 2018.

[44] H. Sajjadi, A. A. Delouei, M. Izadi, and R. Mohebbi, "Investigation of MHD natural convection in a porous media by double MRT lattice Boltzmann method utilizing MWCNT- $\mathrm{Fe}_{3} \mathrm{O}_{4} /$ water hybrid nanofluid," International Journal of Heat and Mass Transfer, vol. 132, pp. 1087-1104, 2019.

[45] T. Muhammad, K. Rafique, M. Asma, and M. Alghamdi, "Darcy-Forchheimer flow over an exponentially stretching curved surface with Cattaneo-Christov double diffusion," Physica A: Statistical Mechanics and its Applications, vol. 556, article 123968, 2020. 
[46] M. A. Sadiq, F. Haider, T. Hayat, and A. Alsaedi, "Partial slip in Darcy-Forchheimer carbon nanotubes flow by rotating disk," International Communications in Heat and Mass Transfer, vol. 116, article 104641, 2020.

[47] M. Alghamdi, A. Wakif, T. Thumma, U. Khan, D. Baleanu, and G. Rasool, "Significance of variability in magnetic field strength and heat source on the radiative-convective motion of sodium alginate-based nanofluid within a Darcy- Brinkman porous structure bounded vertically by an irregular slender surface," Thermal Engineering, vol. 28, article 101428, 2021.

[48] M. Khan, A. M. El Shafey, T. Salahuddin, and F. Khan, "Chemically Homann stagnation point flow of Carreau fluid," Physica A: Statistical Mechanics and its Applications, vol. 551, article 124066, 2020.

[49] G. Rasool, A. Shafiq, M. S. Alqarni, A. Wakif, I. Khan, and M. S. Bhutta, "Numerical scrutinization of DarcyForchheimer relation in convective magnetohydrodynamic nanofluid flow bounded by nonlinear stretching surface in the perspective of heat and mass transfer," Micromachines, vol. 12, no. 4, p. 374, 2021.

[50] H. Waqas, T. Muhammad, S. Hussain, S. Yasmin, and G. Rasool, "Consequences of Fourier's and Fick's laws in bioconvective couple stress nanofluid flow configured by an inclined stretchable cylinder," International Journal of Modern Physics B, vol. 35, no. 17, article 2150176, 2021. 